\title{
Research on energy-efficient routing algorithm based on SWIPT in multi-hop clustered WSN for 5 G system
}

\author{
Shu Han ${ }^{1 *} \mathbb{D}$, Xiao-ming Liu', Hong-yu Huang² ${ }^{1}$ Fei Wang ${ }^{3}$ and Yuan-hong Zhong
}

*Correspondence:
hs@cqu.edu.cn
${ }^{1}$ School of Microelectronics
and Communication
Engineering,
Chongqing University,
Chongqing 400044, China
Full list of author information
is available at the end of the
article

${ }^{*}$ Correspondence:

hs@cqu.edu.cn

and Communication

Engineering,

Chongqing University,

Full list of author information article

\begin{abstract}
As one of the basic supporting technologies of $5 \mathrm{G}$ system, wireless sensor networks technology is facing a new challenge to improve its transmission energy efficiency. This paper considers combining simultaneous wireless information and power transfer (SWIPT) technique and routing technique, and applying them to multi-hop clustered wireless sensor networks (MCWSN), where each node can decode information and harvest energy from a received radio-frequency signal. And the relay nodes in MCWSN can utilize the harvest energy to forward data to their next hop nodes according to the routing scheme. First, we formulate an energy-efficient routing problem of MCWSN with SWIPT. Then, a heuristic energy efficient cooperative SWIPT routing algorithm (EECSR) is presented to find a transmission path with the maximum energy efficiency. Specifically, in EECSR, the resource allocation problem in each hop of the path is transformed to some equivalent convex optimization problems, which are resolved via dual decomposition. Moreover, a distributed routing protocol based on EECSR is proposed. As far as we know, this is the first solution that considers energy efficiency optimization based on routing and SWIPT in MCWSN. Simulation results show that our EECSR algorithm has high energy efficiency and good robustness. And our distributed routing protocol has better real-time performance than traditional protocols.
\end{abstract}

Keywords: SWIPT, Routing, Energy efficiency, Resource allocation, Clustered WSN, $5 \mathrm{G}$ system

\section{Introduction}

At present, 5G communication technologies have successfully achieved high-speed data transmission, and various wireless sensor networks have undertaken the work of collecting front-end data for $5 G$ network $[1,2]$. In other words, WSN is one of the important technologies to realize the industrialization of 5G system. Fortunately, technologies like edge cloud computing [3-5] and heterogeneous network convergence [6-8] provide the possibility for the integration of WSN and 5G high-speed networks. In addition, network slicing technology $[9,10]$ enables $5 G$ network to flexibly provide services for front-end sensor networks in different application scenarios. And the research of radio access network (RAN) slicing technology enables gateway nodes of front-end networks to access the core network efficiently, securely and flexibly [11-13]. In other words, the

C The Author(s) 2021. Open Access This article is licensed under a Creative Commons Attribution 40 International License, which permits use, sharing, adaptation, distribution and reproduction in any medium or format, as long as you give appropriate credit to the original author(s) and the source, provide a link to the Creative Commons licence, and indicate if changes were made. The images or other third party material in this article are included in the article's Creative Commons licence, unless indicated otherwise in a credit line to the material. If material is not included in the article's Creative Commons licence and your intended use is not permitted by statutory regulation or exceeds the permitted use, you will need to obtain permission directly from the copyright holder. To view a copy of this licence, visit http:// creativecommons.org/licenses/by/4.0/. 
development of $5 \mathrm{G}$ system has brought new opportunities for WSN. However, new technologies and applications based on $5 \mathrm{G}$ come with new challenges, which require WSN to have higher transmission energy efficiency and longer life time [14].

Energy limitation has always been one of the challenges of WSN applications. It is well known that most of the energy in wireless networks is consumed in data transmission. Therefore, maximizing transmission energy efficiency is one of the concerns in the application and research of WSN. In recent years, a radio-frequency (RF) based energy harvesting technology, namely, simultaneous wireless information and power transmission (SWIPT), has been regarded as a promising technique to improve transmission energy efficiency of wireless networks $[15,16]$. For WSN, SWIPT can make nodes harvest energy and receive information via the same RF signal [17]. This feature of SWIPT can balance the energy distribution and provide a potential long-term operation of networks. By employing a power splitting (PS) or a time switching (TS) circuit, receivers in SWIPT can adjust the splitting ratio to change the throughput and energy consumption of communication links $[18,19]$.

In recent years, SWIPT has been studied to improve the energy efficiency of different wireless systems related to $5 \mathrm{G}$ network, such as orthogonal frequency-division multiple (OFDM) systems $[15,20,21]$, multiple-input multiple-output (MIMO) systems [7, 18], femtocells [16] and mobile clustered WSN [22]. Moreover, due to the high energy efficiency and reliability, SWIPT cooperative networks have aroused more interest of researchers. For example, in [23], the authors have studied resource allocation policies in the cooperative wireless network with SWIPT to optimize energy efficiency. In [24, 25], the authors have presented the energy efficiency optimization for SWIPT in MIMO two-way amplify-and-forward relay networks. Meanwhile, in order to improve energy and spectrum efficiencies of wireless networks, some studies have considered applying SWIPT to cooperative NOMA wireless networks $[26,27]$. In addition, the relay selection policy in cooperative networks has been studied based on the tradeoff between information transmission and energy harvest [28]. The authors of [29] have formulated a resource allocation problem in wirelessly powered sensor networks, and have presented an energy efficient cooperative transmission scheme based on SWIPT.

It can be observed that most studies on SWIPT technology have only focused on onehop or two-hop small networks. However, in the applications of WSN, multi-relay transmission can effectively reduce the transmit power and improve the efficiency of data collection [30, 31]. Moreover, based on the energy supplement function of SWIPT, some studies have also applied SWIPT to multi-hop wireless networks to improve network performance [32-36]. In [32], the authors have optimized the energy harvest ratios of multi-hop amplify-and-forward (AF) and decode-and-forward (DF) WSN with SWIPT. In a multi-hop SWIPT DF sensor network, a problem of minimizing transmitting energy has been studied under an end-to-end throughput constraint [33]. Subject to transmit power constraints, the authors of $[34,35]$ have focused on the relay beamforming design to maximize the achievable rate of a MIMO multi-hop sensor network with SWIPT. In [36], SWIPT has been applied to a multi-hop clustered WSN, where the transmission energy efficiency sum has been maximized. However, this paper has only focused on the communication between two adjacent cluster heads instead of the source nodes and the sink. 
Most of the above works on SWIPT in multi-hop networks have only considered improving the performance of one given path. The authors of $[37,38]$ have started to study routing algorithms based on SWIPT in multi-hop energy-constrained WSNs. And these two works have shown that routing algorithms based on SWIPT can find the transmission paths with the maximum throughput and the minimum consumption energy, respectively. As we know, routing can affect the energy efficiency of the transmission paths in WSN $[39,40]$. Therefore, in this paper, we consider to supplement the energy of the relays by implementing SWIPT in multi-hop clustered wireless sensor networks (MCWSN). Based on this scheme, we plan to introduce a routing algorithm to further exploit SWIPT. Our approach has advantages as follows: (1) SWIPT allows us to dynamically adjust the data rate and energy consumption of links to achieve higher transmission energy efficiency; (2) Benefiting from the energy supplement of SWIPT, relay selection becomes more flexible; (3) Relays replenished by SWIPT may form a routing scheme with higher transmission energy efficiency.

Although the combination of SWIPT and routing algorithm has the above advantages, it is not easy to combine these two techniques directly. The main challenge of our work is to concurrently consider the impacts of routing and resource allocation on the transmission energy efficiency of a path. Note that the resource mentioned here specifically refers to the transmit power of nodes in paths, the energy harvested at the receivers, and candidate relay nodes. The more challenging thing is that the routing policy and the resource allocation policy interact with each other. On one hand, different routing strategies will make each node in the path have a different next hop node, which will obviously result in different resource allocation policies. On the other hand, in each hop, resource allocation policies can determine the routing metrics of corresponding links and nodes. And different routing metrics may cause different routing results. Therefore, our aim is to find the most energy efficient transmission path for source $\mathrm{CHs}$ by jointly determining the optimal policies, including the optimal routing policy and the resource allocation policy for each hop.

For this purpose, we first formulate an energy-efficient routing problem for multihop clustered wireless sensor networks with PS-SWIPT. Then we design a heuristic energy efficient cooperative SWIPT routing algorithm (EECSR) to decompose our problem into an energy efficient routing subproblem and multiple resource allocation subproblems. In EECSR, during the process of finding the energy efficient paths, the resource allocation policies of communication links are solved. Specifically, the resource allocation subproblems for links are formulated as non-convex optimization problems with the object of maximum energy efficiency. These problems are transformed to equivalent convex problems and then optimally resolved by the dual decomposition method. Furthermore, we provide a table-driven distributed energyefficient cooperative SWIPT routing (TDEECSR) protocol for our routing problem. MCWSN is a type of sensor network often used in object monitoring applications. As far as we know, this is the first work considering the application of SWIPT-based routing algorithms to MCWSN's transmission energy efficiency optimization. Our simulation experiments demonstrate the convergence of proposed algorithms. Then, the impacts of parameters on the energy efficiency of our algorithms are analyzed. Moreover, under different transmission modes and network energy scenarios, a lot of 
simulation results prove the energy efficiency of our EECSR algorithm and the practicability of TDEECSR protocol.

\section{System model}

\subsection{Network model}

In this paper, we consider a multi-hop clustered wireless sensor network (MCWSN) as shown in Fig. 1. The sink node is the data collection end. The nodes in the network are divided into multiple clusters. In a cluster, all member nodes are distributed within onehop communication range of the cluster head $(\mathrm{CH})$, which has better performance than that of the members. $\mathrm{CHs}$ can form a multi-hop path to transmit data from a source $\mathrm{CH}$ to the sink, like the path $1 \rightarrow 2 \rightarrow 3 \rightarrow$ sink in Fig. 1, where $\mathrm{CH}_{1}$ is the source, $\mathrm{CH}_{2}$ and $\mathrm{CH}_{3}$ are relays. In addition, there are some cluster members which can communicate with more than one $\mathrm{CH}$. We define this kind of member nodes as candidate relays for the cooperative transmission between two adjacent $\mathrm{CHs}$, e.g., $r_{12}(1), r_{12}(2)$ and $r_{12}(3)$ in Fig. 1.

We choose power splitting (PS) mode instead of time splitting (TS) mode to support SWIPT. This is because TS mode requires that the transceiver nodes in communication links must be time synchronized. However, this requirement may not be guaranteed in some harsh application environments of WSN. We can observe in Fig. 2 that one node contains a receiving antenna, a PS unit, an energy harvester (EH) circuit, an information decoder (ID) circuit and a rechargeable battery. The node can split the received signal power into two parts according to $\rho^{I}$ and $\rho^{E}$, where $\rho^{I}$ is the power splitting ratio of information decoding, and $\rho^{E}$ is the ratio of energy harvesting. The battery can be recharged by the harvest energy, and can support the information decoding and forwarding. Two kinds of links can work in SWIPT state. One is the link from a source $\mathrm{CH}$ to a member relay, like the link from $\mathrm{CH}_{1}$ to $r_{1,2}(2)$ in Fig. 2. And the other kind is the link between CHs. In these SWIPT links, the receivers can dynamically adjust their power splitting ratios.
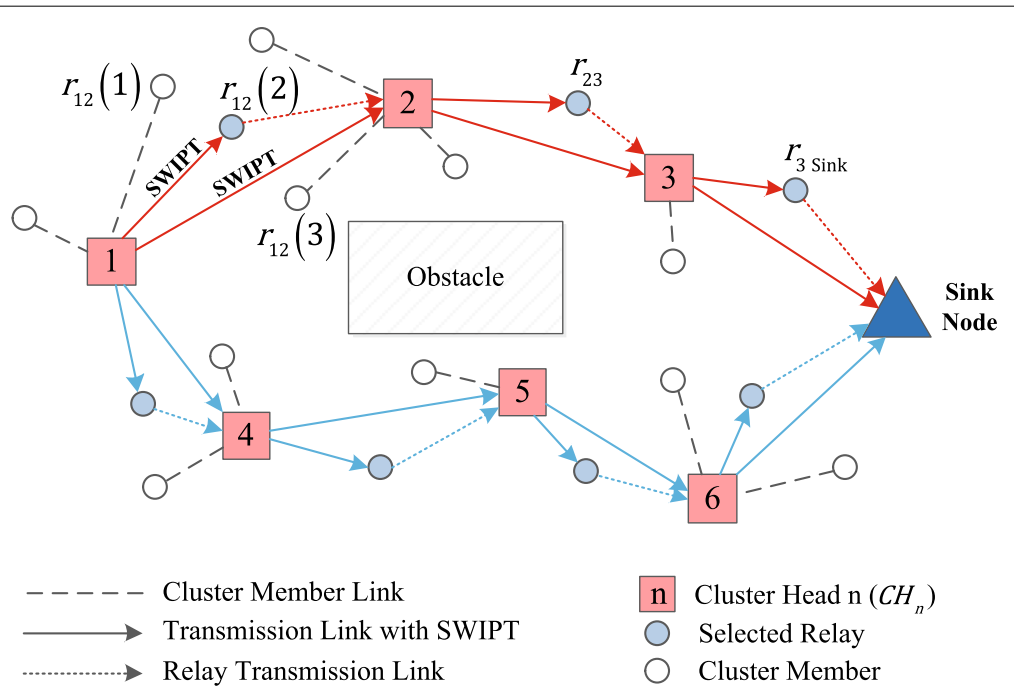

n Cluster Head n $\left(\mathrm{CH}_{n}\right)$

Selected Relay

Cluster Member

Fig. 1 A multi-hop clustered cooperative wireless sensor network with SWIPT 


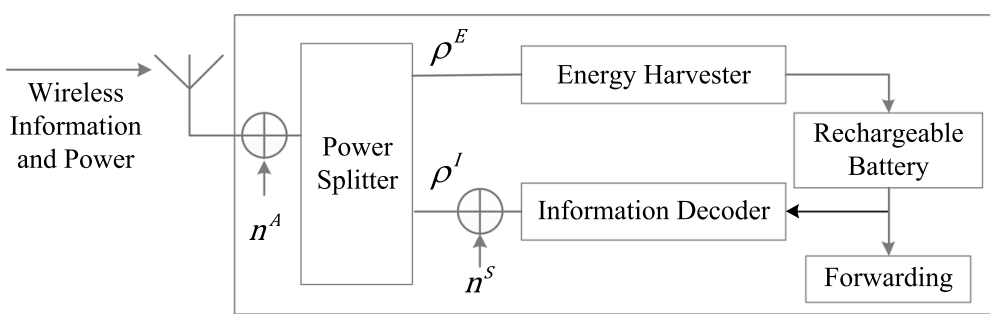

Fig. 2 A diagram of a node with SWIPT

\subsection{Transmission model}

In the network, nodes are half-duplex and channels are symmetrical [15]. If we regard the sink as a special $\mathrm{CH}$, then a multi-hop path is a chain of $\mathrm{CH}$-to- $\mathrm{CH}$ cooperative transmission subnetworks, which we may refer as subnet in the following. In each subnet, we have one source $\mathrm{CH}_{i}$ and one destination $\mathrm{CH}_{j}$, where $i, j \in \mathcal{K}$, and $\mathcal{K}$ is the set of identity number of all $\mathrm{CHs}$. In addition, there are a set of the candidate relays between the source and destination $\mathrm{CHs}$, which is named as relay candidate set $\mathcal{N}_{i j}$. Each subnet contains links such as $l i n k_{i r}, l i n k_{r j}$ and $l i n k_{i j}$, where $r \in \mathcal{N}_{i j}$. Corresponding to these links, we let the channel gains as $h_{i r}, h_{r j}$ and $h_{i j}$, and let the additive white Gaussian noise power be $\sigma_{i j}^{2}, \sigma_{i r}^{2}$ and $\sigma_{r j}^{2}$, respectively. On the premise that the receiver can obtain the perfect channel state information (CSI), the normalized effective channel gains are defined as $g_{i j}=\left|h_{i j}\right|^{2} / \sigma_{i j}^{2}, g_{i j}=\left|h_{i r}\right|^{2} / \sigma_{i r}^{2}, g_{i j}=\left|h_{r j}\right|^{2} / \sigma_{r j}^{2}$, where $h_{a b}=K_{a b} /\left(L_{a b}\right)^{\alpha}, K_{a b}$ characterizes the small-scale Rayleigh fading of the channel between the transmitter $a$ and the receiver $b, L_{a b}$ is the distance between $a$ and $b$, and $\alpha$ is the path loss coefficient.

In a determined routing scheme, the data transmission in one path is implemented by the subnet of each hop. So, we just describe the details of cooperative transmission in one subnet as follows. Before transmission, the source $\mathrm{CH}_{i}$ in a subnet selects a cooperative relay with the highest priority in the $\mathcal{N}_{i j}$. The calculation of the priority of each candidate relay will be introduced in the "Resource allocation optimization for subnetworks" section. We assume that the relays in the network adopt the decode and forward (DF) method. Then a cooperative transmission includes the following two phases.

PHASE 1 The direct transmission. $\mathrm{CH}_{i}$ sends a message signal with power $P_{i}$. Then, with the PS-SWIPT scheme, the signal to noise ratio (SNR) at the $\mathrm{CH}_{j}$ is expressed as

$$
\gamma_{j, 1}=\frac{\rho_{j}^{I} P_{i}\left|h_{i j}\right|^{2}}{\sigma_{i j}^{2}}=\rho_{j}^{I} P_{i} g_{i j},
$$

where the subscript 1 in $y_{j, 1}$ denotes the first phase. Note that signal processing noise at the receiver is $\mathrm{n}_{\mathrm{j}}^{\mathrm{S}} \sim \mathrm{CN}\left(0, \sigma_{\mathrm{ij}}^{2}\right)$. And the antenna noise $\mathrm{n}_{\mathrm{j}}^{\mathrm{A}}$ in Fig. 2 is neglected as it is negligible in comparison with $\mathrm{n}_{\mathrm{j}}^{\mathrm{S}}$ [41]. In addition, the harvested energy at receiver $\mathrm{CH}_{j}$ is given by

$$
E_{i j}=\eta \rho_{j}^{E}\left|h_{i j}\right|^{2} P_{i} \cdot \frac{T}{2}
$$


where $T$ is the total time for the cooperative transmission, and the first phase occupies a half of $T . \eta$ denotes the energy harvesting coefficient with $0<\eta<1$. Similarly, The SNR at $r$ can be expressed as

$$
\gamma_{r}=\frac{\rho_{r}^{I} P_{i}\left|h_{i r}\right|^{2}}{\sigma_{i r}^{2}}=\rho_{r}^{I} P_{i} g_{i r}
$$

PHASE 2 The relay transmission. The relay $r$ forwards the decoded message to $\mathrm{CH}_{j}$. We assume that all the energy harvested by the relay $r$ in PHASE 1 is used for its forwarding in PHASE 2. So, the SNR at $\mathrm{CH}_{j}$ in PHASE 2 can be expressed as

$$
\gamma_{j, 2}=\frac{P_{i} \eta \rho_{r}^{E}\left|h_{i r}\right|^{2}\left|h_{r j}\right|^{2}}{\sigma_{r j}^{2}}=P_{i} \eta \rho_{j}^{I} \rho_{r}^{E}\left|h_{i r}\right|^{2} g_{r j} .
$$

At the end of PHASE 2, $\mathrm{CH}_{j}$ uses maximum ratio combining (MRC) to combine the signals from $\mathrm{CH}_{i}$ and $r$. Thus, the equivalent received SNR of $\mathrm{CH}_{j}$ is given by

$$
\gamma_{j}=\rho_{j}^{I} P_{i} g_{i j}+P_{i} \eta \rho_{j}^{I} \rho_{r}^{E}\left|h_{i r}\right|^{2} g_{r j}
$$

It can be seen from (5) that the cooperative relay helps to increase the equivalent SNR of $\mathrm{CH}_{j}$. Therefore, the achievable data rate from $\mathrm{CH}_{i}$ to $\mathrm{CH}_{j}$ with selected relay $\mathrm{r}$ is given by

$$
R_{i j}=\frac{B}{2} \log _{2}\left(1+\min \left\{\gamma_{r}, \gamma_{j}\right\}\right)
$$

where $B$ is the bandwidth of the baseband. Since the entire transmission process is divided into two phases with equal time, the data rate is divided by 2 . Observing (6), we can find that when $\gamma_{r}$ is equal to $\gamma_{j}$, the maximum transmission rate can be achieved. So, we use the equation $\gamma_{r}=\gamma_{j}$ as a condition to derive $\rho_{r}^{I}$ of $r$. We let $\rho_{r}^{E}=1-\rho_{r}^{I}$. Then we obtain

$$
\rho_{r}^{I}=\frac{\rho_{j}^{I}\left(g_{i j}+g_{r j} \eta\left|h_{i r}\right|^{2}\right)}{g_{i r}+\rho_{j}^{I} g_{r j} \eta\left|h_{i r}\right|^{2}} .
$$

We assume that $\rho_{r}^{E}=1-\rho_{r}^{I}$, which means that $\rho_{r}^{I}$ of relay is first determined by the ID unit in Fig. 2 to satisfy maximum energy efficiency, then all the remaining power flow is allocated to the EH unit to support relay transmission. Based on (6) and (7), the achievable data rate from $\mathrm{CH}_{i}$ to $\mathrm{CH}_{j}$ with relay $r$ is given by

$$
\begin{aligned}
& R_{i j}^{r}=\frac{B}{2} \log _{2}\left(1+\lambda_{i j}^{r} P_{i j}^{r}\right), \\
& \lambda_{i j}^{r}=\rho_{r}^{I} g_{i r}=\frac{\rho_{j}^{I(r)}\left(g_{i j}+g_{r j} \eta\left|h_{i r}\right|^{2}\right) g_{i r}}{g_{i r}+\rho_{j}^{I(r)} g_{r j} \eta\left|h_{i r}\right|^{2}}, \quad r \in \mathcal{N}_{i j},
\end{aligned}
$$

where $\lambda_{i j}^{r}$ and $P_{i j}^{r}=P_{i}$ are the equivalent channel gain and the transmit power, respectively. As shown in (8), we can regard a $\mathrm{CH}_{i}$-to- $\mathrm{CH}_{j}$ subnet as a cooperative transmission link, which may be referred as CoLink $k_{i j}$, in the following. 


\section{Problem formulation}

In this section, we formulate a routing problem with SWIPT in MCWSNs, and find the maximum energy efficiency path from one source $\mathrm{CH}$ to the sink.

\subsection{Energy efficiency cost}

We define the throughput of a subnet as the sum of data bits effectively transmitted to the receiver $\mathrm{CH}$ with each possible relay selection policy. The throughput of the $\mathrm{CH}_{i}$-to$\mathrm{CH}_{j}$ subnet (also named CoLink $k_{i j}$ ) is given by

$$
U_{(i j)}(\mathcal{P}, \rho, \mathcal{S})=\sum_{r=1}^{N_{i j}} \alpha_{r} s_{r} R_{i j}^{r},
$$

where $R_{i j}^{r}$ is defined by (8), $\mathcal{P}=\left\{P_{i j}^{r} \geq 0, r \in \mathcal{N}_{i j}\right\}$ is the power allocation policy, $\rho=\left\{\rho_{j}^{I(r)}, \rho_{j}^{E(r)}, \rho_{r}^{I}, \rho_{r}^{E} \in[0,1], r \in \mathcal{N}_{i j}\right\}$ represents the power splitting policy, and $\mathcal{S}=\left\{s_{r} \in\{0,1\}, r \in \mathcal{N}_{i j}\right\}$ is the relay node selection policy. $s_{r}=1$ means that node $\mathrm{r}$ is selected as the relay, and $s_{r}=0$ means the opposite. $\alpha_{r}$ is a non-negative weight. In addition, we define the power consumption as

$$
U_{T P(i j)}(\mathcal{P}, \boldsymbol{\rho}, \mathcal{S})=\sum_{r=1}^{N_{i j}} \varepsilon s_{r} P_{i j}^{r}+P_{C},
$$

where $P_{C}$ denotes the total static power consumption of the circuits in the subnet; the first term indicates the transmit power consumption of the source cluster head $\mathrm{CH}_{i}$; $\varepsilon \geq 1$ is the conversion efficiency constant of the power amplifier in $\mathrm{CH}_{i}$. Note that the energy harvested by destination $\mathrm{CH}$ is not subtracted from $U_{T P(i j)}(\mathcal{P}, \rho, \mathcal{S})$ as compensation energy. Because, from the perspective of the entire network, the harvested energy is only transferred from node to node to achieve an energy balance. Accordingly, as shown in (11), the energy efficiency of the subnet is defined as the ratio of transmission throughput in bits to per Joule energy consumption.

$$
U_{e f f(i j)}(\mathcal{P}, \rho, \mathcal{S})=\frac{U_{(i j)}(\mathcal{P}, \rho, \mathcal{S})}{U_{T P(i j)}(\mathcal{P}, \rho, \mathcal{S})}
$$

Then, we define the reciprocal of $U_{e f f(i j)}$ in (11) as the energy efficiency cost (EEcost) of the subnet. We can regard EEcost as the price of energy required by sending unit data. And the EEcost of a CoLink is expected to be as small as possible. Furthermore, we define the equivalent energy-efficient cost of a path from the source $\mathrm{CH}_{s c}$ to the sink as

$$
E E \operatorname{cost}_{s c}=\frac{U_{T P}(s c)}{U_{(s c)}}=\frac{\sum_{l_{i j}} U_{T P(i j)}}{\min \left\{U_{(i j)}\right)}, \quad l_{i j} \in \operatorname{path}(s c, \sin k), i, j \in \mathcal{K}
$$

where $l_{i j}$ are links belong to the $p a t h(s c, \operatorname{sink})$. This definition is reasonable, because the maximum throughput of a path is determined by the link with the smallest throughput, and the energy consumption of a path should be the sum of the consumed energy of links. In addition, we define a binary variable $t_{i j}$, with value 1 means that the $\operatorname{link}_{i j}$ is 
selected in the path, and with value 0 means the opposite. So, the energy-efficient cost with routing policy is given as

$$
\widetilde{E_{E \operatorname{cost}}{ }_{s c}}=\frac{\Sigma_{l_{i j}} t_{i j} U_{T P(i j)}}{\min \left\{\frac{U_{(i j)}}{t_{i j}}\right\}}, i, j \in \mathcal{K}, t_{i j} \in\{0,1\} .
$$

\subsection{Optimization problem formulation}

In order to find the maximum efficiency path from a source $\mathrm{CH}$ to the sink, we set the minimum path energy efficiency cost as the optimization object. And the optimization variables are the power allocation policy $\overline{\mathcal{P}}$, the power splitting policy $\overline{\boldsymbol{\rho}}$, the relay node selection policy $\overline{\mathcal{S}}$, and the routing policy $\overline{\mathcal{T}}=\left\{t_{i j} \in\{0,1\}, \quad i, j \in \mathcal{K}\right\}$. The SWIPT routing problem in our network can be formulated as

$$
\text { OPT }-1 \quad \min _{\overline{\mathcal{P}}, \overline{\boldsymbol{\rho}}, \overline{\mathcal{S}}, \overline{\mathcal{T}}} \widetilde{E E \operatorname{cost}} s c(\overline{\mathcal{P}}, \overline{\boldsymbol{\rho}}, \overline{\mathcal{S}}, \overline{\mathcal{T}}),
$$

subject to

$$
\begin{aligned}
& \mathrm{C} 1: \quad E_{i j}^{r} \geq E_{j}^{R e q}, E_{j}^{R e q}=\max \left\{0,\left(E_{j}^{F w d}-E_{C, j}\right)\right\} \quad i, j \in \mathcal{K}, r \in \mathcal{N}_{i j}, \\
& \text { C2: } \quad E_{j}^{F w d}=\frac{T}{2} \sum_{k \in \mathcal{K}} \sum_{r=1}^{N_{j k}} t_{j k} \varepsilon s_{r(j k)} P_{j k}^{r}, j \neq k, j \in \mathcal{K}, r \in \mathcal{N}_{j k}, \\
& \text { C3: } \quad \sum_{j \in \mathcal{K}} \sum_{r=1}^{N_{i j}} t_{i j} s_{r(i j)} P_{i j}^{r} \leq P^{\max }, i \neq j, i \in \mathcal{K}, r \in \mathcal{N}_{i j} \text {, } \\
& \text { C4: } \quad \sum_{i \in \mathcal{K}} \sum_{r=1}^{N_{i j}} t_{i j} s_{r(i j)} R_{i j}^{r} \geq R^{\min }, i \neq j, j \in \mathcal{K}, r \in \mathcal{N}_{i j} \text {, } \\
& \text { C5: } t_{i j} \in\{0,1\}, \quad i, j \in \mathcal{K} \text {, } \\
& \text { C6: } \quad \sum_{j \in \mathcal{K}} t_{j i} \leq 1, C 7: \sum_{j \in \mathcal{K}} t_{i j} \leq 1, \quad i \neq j, i \in \mathcal{K}, \\
& \text { C8: } \\
& \sum_{j \in \mathcal{K}} t_{i j}-\sum_{j \in \mathcal{K}} t_{j i}=\left\{\begin{array}{cc}
1, & i \text { is the source } C H \\
-1, & i \text { is the sink } \\
0, & \text { others in } \mathcal{K}
\end{array}\right. \\
& \text { C9: } \quad s_{r(i j)} \in\{0,1\}, \quad \mathrm{C} 10: \sum_{r=1}^{N_{i j}} s_{r(i j)}=1, \quad i, j \in \mathcal{K}, r \in \mathcal{N}_{i j}, \\
& \text { C11: } \quad \rho_{\min }^{E} \leq \rho_{i j}^{E(r)} \leq \rho_{\max }^{E}, \quad \mathrm{C} 12: \rho_{\min }^{I} \leq \rho_{i j}^{I(r)} \leq \rho_{\max }^{I}, \quad i, j \in \mathcal{K}, r \in \mathcal{N}_{i j} \text {, } \\
& \text { C13: } \quad \rho_{i j}^{I(r)}+\rho_{i j}^{E(r)} \leq 1, \quad i, j \in \mathcal{K}, r \in \mathcal{N}_{i j} \text {, }
\end{aligned}
$$

where $C 1$ is the energy harvesting constraint; $E_{i j}^{r}$ is defined by (2); $E_{j}^{R e q}$ is the energy required by the receiver $\mathrm{CH}_{j}$ for data forwarding, which is defined as $E_{j}^{R e q}=\max \left\{0,\left(E_{j}^{F w d}-E_{C, j}\right)\right\}, E_{j}^{F w d}$ is the transmit energy of $C H_{j}$ for forwarding, and $E_{C, j}$ is the remaining battery energy of $\mathrm{CH}_{j}$. Obviously,if $E_{j}^{F w d}<E_{C, j}, C 1$ becomes $E_{i j}^{r} \geq 0$ so that the energy constraint is relaxed. $C 3$ gives the transmit power constraint. The maximum transmit power $P_{i}^{\max }$ is determined by the battery capacity $E_{i}^{B}$. $C 4$ is the transmission data rate constraint which requires that all subnets in the path solution should be greater than $R^{\min }$. $C 5-C 8$ are routing constraints which indicate that any node in the path can only have one input CoLink and one output CoLink. In particular, the source node has no input, and the sink node has no output. These constraints can prevent loops and ensure flow conservation. $C 9$ and $C 10$ are cooperative relay 
constraints which indicate that only one node in $N_{i j}$ can be selected. $C 11$ gives the constant lower bound $\rho_{\min }^{E}$ and the upper bound $\rho_{\max }^{E}$ of the power splitting ratio, which are decided by the capability of the receiver. Similarly, $\rho_{\max }^{I}$ and $\rho_{\min }^{I}$ in $C 12$ denote the bounds of the ratio for information decoding. Note that $\rho_{\max }^{E}+\rho_{\min }^{I}=1$ and $\rho_{\text {max }}^{I}+\rho_{\min }^{E}=1$. C13 comes from the power characteristic of the PS unit in Fig. 2, that is, the sum of the splitting power sub-items will never exceed the total received energy.

There are two key challenges in solving OPT-1. The first challenge is that the routing policy can't be exploited directly and the reason is as follows. As shown in constraint $C 1$, the low-limit of the harvest energy of the receiver in a subnet is determined by its $E_{C, j}$ and $E_{j}^{F w d}$. However, the constraint $C 2$ shows that $E_{j}^{F w d}$ depends on the next hop selection policy $t_{j k}$ and the corresponding resource allocation policy in CoLink $k_{j k}$. It means that the change of routing policy $\overline{\mathcal{T}}$ may cause the change of constraint $C 1$ and make the optimization problem more complicated. The second challenge is that the integer constraints $C 5$ and $C 9$ make OPT- 1 become a mixed integer programming problem, which is in general non-convex and NP-hard [22]. Therefore, to address OPT-1, we propose a heuristic routing algorithm to solve this problem.

\section{Problem solution}

\subsection{Energy-efficiency cooperative SWIPT routing algorithm}

We propose a heuristic energy efficient cooperative SWIPT routing algorithm (EECSR) to solve the problem OPT-1. This algorithm takes EEcost as the routing metric, and combines the resource allocation algorithm to calculate EEcosts of CoLinks and CHs. Based on the metrics, EECSR uses a greedy strategy to update the routing policy of each $\mathrm{CH}$. And EECSR is designed based on the framework of the Dijkstra routing algorithm [42]. The algorithm is shown as Table 1.

In EECSR algorithm, $N e x t_{i}$ records the next hop cluster head of $\mathrm{CH}_{i} . E_{i}^{\text {Req }}$ records the harvest energy required by $\mathrm{CH}_{i}$ for forwarding. We assume that the nodes in set $\bar{S}$ have already found the path with the minimum EEcost, while the nodes in set $Q$ haven't found yet. The main body of EECSR is a while loop in lines 5-17. This loop sequentially adds the node with the smallest $E E$ cost in $Q$ to $\bar{S}$, and then update the costs of related neighbors, as shown in lines $8-16$. When all the nodes in $Q$ are added to $\bar{S}$, the loop ends. Concretely, in line 9, the first inequality shows that if $\mathrm{CH}_{i}$ chooses $\mathrm{CH}_{j}$ as its next hop node, EEcost ${ }_{i}$ may become smaller. In the second inequality, for $\mathrm{CH}_{j}$ in $\operatorname{CoLink}_{i j}$, the maximum harvest energy $E_{i j}^{\max }=\frac{T}{2} \eta\left|h_{i j}\right|^{2} P^{\max }$ should be greater than the required forwarding energy. If both inequalities are true, EECSR calls a sub-algorithm to solve the resource allocation problem of CoLink $k_{i j}$, as shown in line 10. Specifically, the input of this sub-algorithm includes $h_{i j}, \sigma_{i j}, h_{i r}, \sigma_{i r}, h_{r j}, \sigma_{r j}, P^{\max }, R^{\min }, \eta, T, \alpha_{r}, \varepsilon$ and $P_{C}$. The output of the sub-algorithm includes $P_{i j}^{r}, \rho_{j}^{E(r)}, \rho_{j}^{I(r)}, \rho_{r}^{E}, \rho_{r}^{I}$ and $s_{r}$. In addition, the sub-algorithm also obtains $E E \operatorname{cost}_{(i j)}^{*}, U_{(i j)}^{*}, U_{T P(i j)}^{*}$ of $\operatorname{CoLink}_{i j}$. Note that in the sub-algorithm, we introduce $E_{j}^{\text {Req }}$ to characterize the impact of the receiver's routing policy. According to (12), we can calculate the $E E \operatorname{cost}_{i}^{\prime}, U_{T P(i)}^{\prime}, U_{(i)}^{\prime}$ in line 11 as 
Table 1 Energy-efficiency cooperative SWIPT routing algorithm

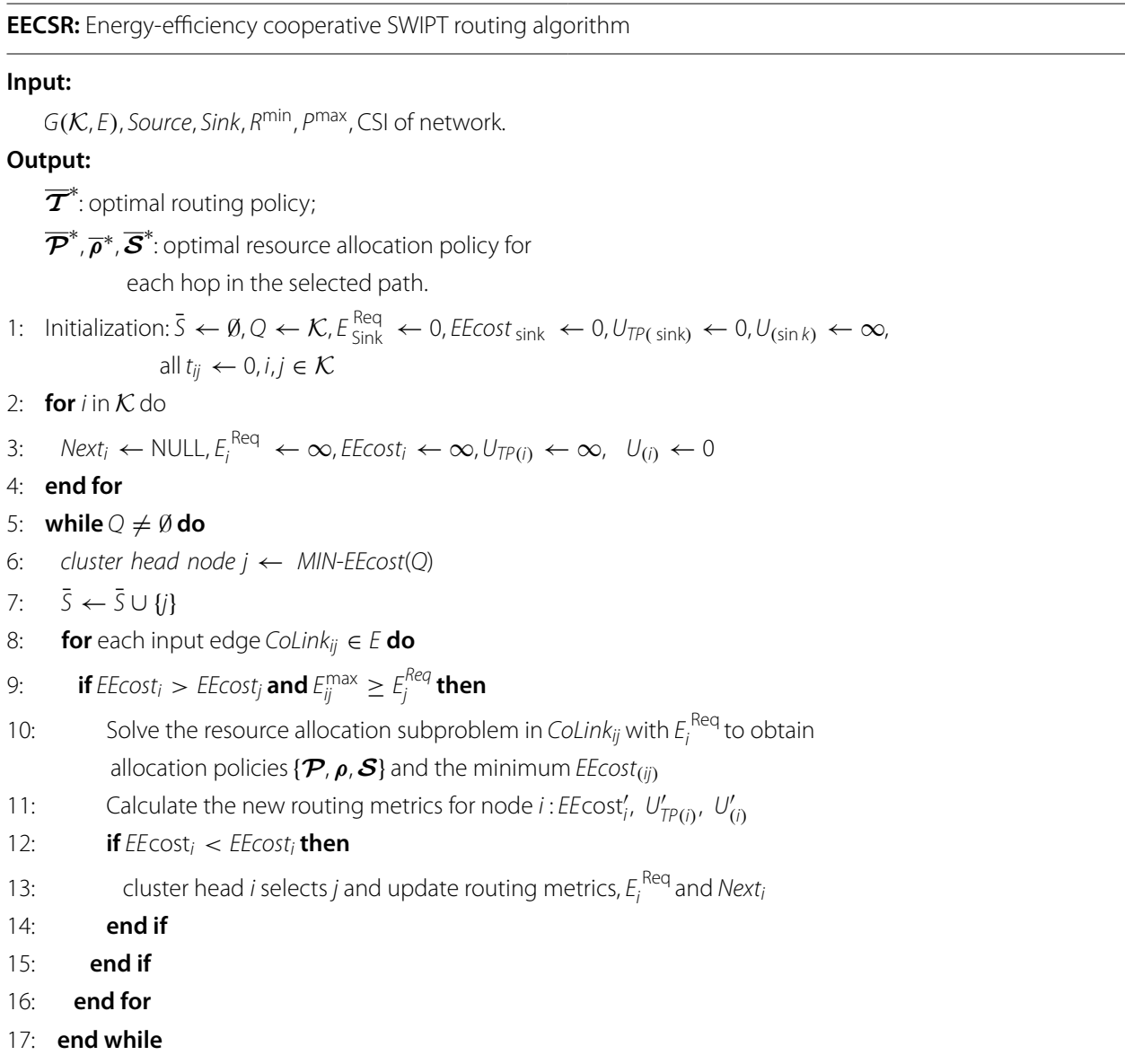

$$
\mathrm{EEcost}_{i}^{\prime}=\frac{U_{T P(i)}^{\prime}}{U_{(i)}^{\prime}}=\frac{U_{T P(i j)}^{*}+U_{T P(j)}}{\min \left\{U_{(i j)}^{*}, U_{(j)}\right\}} .
$$

In lines 12 and 13, if $E E \operatorname{cost} t_{i}^{\prime}$ is lower than $E E \operatorname{cost} t_{i}$, the values of $E E \operatorname{cost} t_{i}, U_{(i)}, U_{T P(i)}$ are updated to $E E \cos t_{i}^{\prime}, U_{(i)}^{\prime}, U_{T P(i)}^{\prime}$. And $E_{i}^{R e q}$ is updated according to the definition of the $E_{j}^{R e q}$ in constraint $C 1$ of OPT-1. Next $t_{i}$ is updated to $j$. Obviously, once EECSR runs over, the routing policy $\overline{\mathcal{T}}^{*}$ for all $\mathrm{CHs}$ is determined.

In summary, EECSR decomposes OPT-1 into an outer routing subproblem and multiple inner resource allocation subproblems. Once node $j$ joins $\bar{S}$, its routing policy will never change, so the constraints $C 1$ and $C 2$ of OPT-1 for CoLink $i j$ are determined. Since the forward routing policies and energy consumption requirements of the receivers are known, we can solve the resource allocation problems for subnets independently. 


\subsection{Resource allocation optimization for subnetworks}

In this subsection, the resource allocation subproblem in line 10 of EECSR is modeled first. Then, an iterative sub-algorithm is presented to solve these subproblems. And in each iteration of the sub-algorithm, an equivalent convex optimization problem is resolved via dual decomposition.

\subsubsection{Resource allocation optimization for subnetworks}

For one subnet, we formulate the inner resource allocation subproblem in EECSR as (15), where the optimization goal is the minimum EEcost, and the optimization variables $\mathcal{P}, \rho, \mathcal{S}$ determine the power allocation, power splitting and relay selection policies, respectively.

The subscripts $i$ and $j$ in OPT-2 are fixed, which represent the sender and receiver $\mathrm{CHs}$ of the given $\mathrm{CoLink}_{i j}$, respectively. Therefore, although the constraints $C 1, C 2, C 3, C 4-C 8$ in OPT-2 have similar physical meanings as $C 1, C 3, C 4, C 9-C 13$ in OPT-1, the dimensions of the variables are all reduced from $\left(i \times j \times N_{i j}\right)$ to $N_{i j}$ in OPT-2. Then, we can observe from (15) that there are still some obstacles in solving OPT-2. First, the objective function of OPT-2 is not convex with respect to $\mathcal{P}, \rho$ and $\mathcal{S}$. Second, optimization variables $\rho$ and $\mathcal{P}$ are coupled in the objective function and $C 1, C 3$ in OPT-2. Third, the integer constraint for relay selection in $C 4$ makes constraints can't span a convex set. In order to make OPT-2 tractable, we do some equivalent transformation and relaxation operations as described belows.

$$
O P T-2 \quad \min _{\mathcal{P}, \rho, \mathcal{S}} E E_{\operatorname{cost}}(i j)(\mathcal{P}, \rho, \mathcal{S}),
$$

subject to

$$
\begin{array}{ll}
\mathrm{C} 1: & E_{i j}^{r} \geq E_{j}^{\mathrm{Req}}, \quad r \in \mathcal{N}_{i j}, \\
\mathrm{C} 2: & \sum_{N_{i j}}^{N_{i j}} s_{r} P_{i j}^{r} \leq P^{\max }, \\
\mathrm{C} 3: & \sum_{r=1}^{r=1} s_{r} R_{i j}^{r} \geq R^{\min }, \\
\mathrm{C} 4: & s_{r} \in\{0,1\}, r \in \mathcal{N}_{i j}, \quad \text { C5: } \quad \sum_{r=1}^{N_{i j}} s_{r}=1, \\
\mathrm{C} 6: & \rho_{\min }^{E} \leq \rho_{j}^{E(r)} \leq \rho_{\max }^{E}, \quad \mathrm{C} 7: \quad \rho_{\min }^{I} \leq \rho_{j}^{I(r)} \leq \rho_{\max }^{I}, \quad r \in \mathcal{N}_{i j}, \\
\mathrm{C} 8: \quad \rho_{j}^{I(r)}+\rho_{j}^{E(r)} \leq 1, \quad r \in \mathcal{N}_{i j} .
\end{array}
$$

\subsubsection{Algorithm for resource allocation problem}

For convenience, we first equivalently transform the optimization objective in (15) to the maximum $U_{e f f(i j)}$. Then we observe that the fractional form of the $U_{\text {eff }(i j)}$ still makes OPT-2 a nonlinear programming problem. Similar to $[15,36]$, we use Dinkelbach method [43] to convert the objective function into an equivalent subtractive form as follows

$$
F(\mathcal{P}, \rho, \mathcal{S})=U_{(i j)}(\mathcal{P}, \rho, \mathcal{S})-q^{*} U_{T P(i j)}(\mathcal{P}, \rho, \mathcal{S})
$$

where $q^{*}$ is defined as the maximum energy efficiency of a CoLink and is given as 


$$
q^{*}=\frac{U_{(i j)}\left(\mathcal{P}^{*}, \boldsymbol{\rho}^{*}, \mathcal{S}^{*}\right)}{U_{T P(i j)}\left(\mathcal{P}^{*}, \boldsymbol{\rho}^{*}, \mathcal{S}^{*}\right)}=\max _{\mathcal{P}, \boldsymbol{\rho}, \delta} U_{e f f(i j)}(\mathcal{P}, \boldsymbol{\rho}, \mathcal{S}) .
$$

The equivalence of the above transformation is supported by the following theorem [44].

Theorem 1 The maximum energy efficiency $q^{*}$ is achieved if and only if

$$
\begin{gathered}
\max _{\mathcal{P}, \boldsymbol{\rho}, \mathcal{S}} F(\mathcal{P}, \boldsymbol{\rho}, \mathcal{S})=F\left(\mathcal{P}^{*}, \boldsymbol{\rho}^{*}, \mathcal{S}^{*}\right)=0 \\
\operatorname{for} U_{(i j)}(\mathcal{P}, \boldsymbol{\rho}, \mathcal{S})>0 \text { and } U_{T P(i j)}(\mathcal{P}, \boldsymbol{\rho}, \mathcal{S})>0 .
\end{gathered}
$$

Brief Proof: According to (9) and (10), $U_{(i j)}(\mathcal{P}, \rho, \mathcal{S})$ and $U_{T P(i j)}(\mathcal{P}, \rho, \mathcal{S})$ are positive. In addition, $F(\mathcal{P}, \rho, \mathcal{S})$ and $q^{*}$ are defined in (16). Then, following the similar method in [43], we can complete the rest of the proof.

Similar to the method in $[15,36]$, we propose an energy-efficient subnet resource allocation algorithm (EESRA) to solve OPT-2, as shown in Table 2.

According to line 3 of EESRA, we solve the problem (17) in each iteration of the while loop.

$$
O P T-3 \max _{\mathcal{P}, \rho, \mathcal{S}} F(\mathcal{P}, \rho, \mathcal{S})
$$

subject to $\quad C 1-C 8$ in OPT-2 .

Table 2 Energy efficient subnet resource allocation algorithm

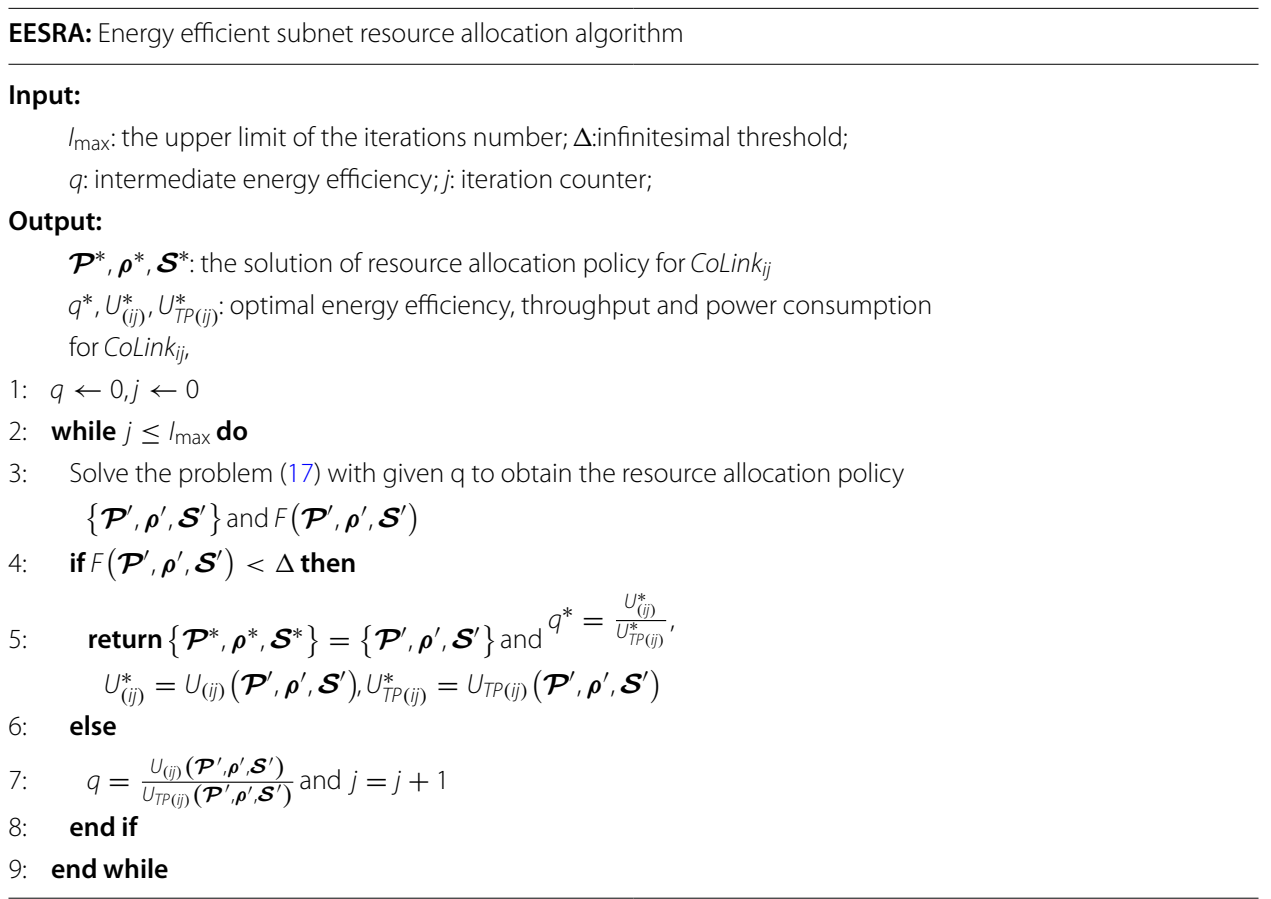


For solving the problem (17), we have the following results.

Theorem 2 EESRA can converge to the maximum $U_{\text {eff }(i j)}$ by solving OPT-3 in each iteration.

\section{Brief Proof:}

The outline of the method is as follows. First, we adopt similar methods in [43, 44] to prove the following three propositions: (1) $\bar{F}(q)$ is strictly monotonically decreasing with respect to $q$, where $\bar{F}(\cdot)$ is the maximum $F(\mathcal{P}, \rho, \mathcal{S})$ with a given $q$ in (11); (2) For any value of $q$, $\bar{F}(q)$ is always greater than or equal to zero; (3) q increases in each iteration. By combining above three propositions, we can prove that after enough iterations, $\bar{F}(q)$ will converge to zero and satisfy the optimality condition in Theorem 1.

In the following steps, we transform OPT-3 into a convex optimization problem. First, we use the time-sharing relaxation technique [45] to handle the integer constraint and relax the $s_{r}$ to a real number from 0 to 1 in C4 of OPT-3. In fact, we will see in "Dual decomposition solution" section that although relaxation is done here, the optimal $s_{r}$ will still remain Boolean. Second, we define two auxiliary variables as $\tilde{P}_{i j}^{r}=s_{r} P_{i j}^{r}$ and $\tilde{\rho}_{j}^{I(r)}=s_{r} \rho_{j}^{I(r)}$, which can be interpreted as the actual transmit power of the sender and the actual splitting ratio of the receiver, respectively. In addition, we replace $R_{i j}^{r}$ in the objective function and $C 3$ in (17) by $\tilde{R}_{i j}^{r}=\frac{B}{2} \log _{2}\left(\tilde{\lambda}_{i j}^{r} \frac{\tilde{P}_{i j}^{r}}{s_{r}}\right)$, where $\tilde{\lambda}_{i j}^{r}=\frac{\frac{\tilde{\rho}_{j}^{I(r)}}{s_{r}}\left(g_{i j}+g_{r i} \eta\left|h_{i r}\right|^{2}\right) g_{i r}}{g_{i r}+\frac{\tilde{\rho}_{j}^{I(r)}}{s_{r}} g_{r j} \eta\left|h_{i r}\right|^{2}}$. Third, we decouple $P_{i j}^{r}$ and $\rho_{j}^{E}(r)$ in the constraint $C 1$ in (17) by dividing $\rho_{j}^{E}(r)$ at both sides of the inequality. Then we can rewrite $C 1$ as $C 1^{\prime}: \quad \frac{T}{2} \eta\left|h_{i j}\right|^{2} \tilde{P}_{i j}^{r} \geq \frac{E_{j}^{R e q}}{\rho_{j}^{E(r)}}, \quad r \in \mathcal{N}_{i j}$

Based on the above transformation, we can prove that the transformed OPT-3 problem with constraints $C 1^{\prime}-C 8$ is convex with respect to the $\tilde{P}_{i j}^{r}, \tilde{\rho}_{j}^{I(r)}, \rho_{j}^{E}(r)$ and $s_{r}$. Its specific proof is in Appendix A. The convexity reveals that the transformed OPT-3 problem satisfies Slater's constraint [46]. Therefore, as the optimal duality gap is zero, we design a primal-dual algorithm to solve the transformed OPT-3 problem.

\subsubsection{Dual decomposition solution}

In this subsection, we use Lagrange dual decomposition method [47] to solve the transformed OPT-3 problem. To achieve this goal, we first give the Lagrangian of the transformed OPT-3 by (18), where $\boldsymbol{\beta}=\left[\beta_{1}, \beta_{2}, \ldots, \beta_{N_{i j}}\right]$ is a vector of Lagrange multiplier corresponding to the energy harvesting constraint $C 1^{\prime}$, and $\mathcal{N}_{i j}$ is the cardinality of $\mathcal{N}_{i j}$. Similarly, we introduce Lagrange multipliers $\delta$ for constraint $C 2, \zeta$ for constraint $C 3$, and $\theta$ for constraint $C 4$, respectively. And the Lagrange multiplier corresponding to the power splitting constraint $C 8$ is the vector $\boldsymbol{\mu}=\left[\mu_{1}, \mu_{2}, \ldots, \mu_{N_{i j}}\right]$. In addition, when deriving the power splitting variables, the boundary constraints $C 6$ and $C 7$ can be captured by the Karush-Kuhn-Tucker (KKT) conditions. 


$$
\begin{aligned}
& L(\boldsymbol{\beta}, \delta, \zeta, \theta, \boldsymbol{\mu}, \mathcal{P}, \boldsymbol{\rho}, \boldsymbol{\delta}) \\
& =\sum_{r=1}^{N_{i j}} \alpha_{r} s_{r} \tilde{R}_{i j}^{r}-q\left(\sum_{r=1}^{N_{i j}} \varepsilon \tilde{P}_{i j}^{r}+P_{C}\right) \\
& \quad+\sum_{r=1}^{N_{i j}} \beta_{r}\left(\eta\left|h_{i j}\right|^{2} \tilde{P}_{i j}^{r} \frac{T}{2}-\frac{E_{j}^{R e q}}{\rho_{j}^{E(r)}}\right)- \\
& \delta\left(\sum_{r=1}^{N_{i j}} \tilde{P}_{i j}^{r}-P^{\max }\right)+\zeta\left(\sum_{r=1}^{N_{i j}} s_{r} \tilde{R}_{i j}^{r}-R^{\min }\right)- \\
& \theta\left(\sum_{r=1}^{N_{i j}} s_{r}-1\right)-\sum_{r=1}^{N_{i j}} \mu_{r}\left(\tilde{\rho}_{j}^{I(r)}+\rho_{j}^{E(r)}-1\right) .
\end{aligned}
$$

Then, the dual problem for transformed OPT-3 problem is

$$
\min _{\boldsymbol{\beta}, \delta, \zeta, \theta, \boldsymbol{\mu}} \max _{\mathcal{P}, \boldsymbol{\rho}, \boldsymbol{s}} L(\boldsymbol{\beta}, \delta, \zeta, \theta, \boldsymbol{\mu}, \mathcal{P}, \boldsymbol{\rho}, \boldsymbol{S}) .
$$

We solve this dual problem iteratively in two levels. The lower level is the internal maximization which contains $N_{i j}$ subproblems with identical structure. And the upper level is the external minimization. In each iteration, $N_{i j}$ subproblems are solved for fixed Lagrange multipliers in the lower level. And the solutions of the subproblems are used to update the multipliers in the upper level.

Lower Level: Conventional convex optimization methods and KKT conditions are used to solve sub-problems. For a fixed $q$ in EESRA, the solutions of $\tilde{P}_{i j}^{r}$ and $\tilde{\rho}_{j}^{I(r)}$ for CoLink $k_{i j}$ are given by

$$
\begin{aligned}
& \tilde{P}_{i j}^{r *}=s_{r} P_{i j}^{r *}=s_{r}\left[\frac{B\left(\alpha_{r}+\zeta\right)}{2 \ln (2)\left(q \varepsilon+\delta-\beta_{r} \eta\left|h_{i j}\right|^{2} \frac{T}{2}\right)}\right]_{0}^{p^{\max }}, r \in \mathcal{N}_{i j}, \\
& \tilde{\rho}_{j}^{l(r) *}=s_{r} \rho_{j}^{l(r) *}=s_{r}\left[\frac{\Psi_{r}-g_{i r}}{2 g_{r j} \eta\left|h_{i r}\right|^{2}}\right]_{\rho_{\min }^{I}}^{\rho_{\max }^{l}}, \quad r \in \mathcal{N}_{i j}, \\
& \Psi_{r}=\sqrt{g_{i r}^{2}+\frac{2 g_{r i} g_{i r} \eta\left|h_{i r}\right|^{2} B\left(\alpha_{r}+\zeta\right)}{\ln (2) \mu_{r}}} \\
& \rho_{j}^{E(r)}=\left[\sqrt{\frac{\beta_{r} E_{j}^{R e q}}{\mu_{r}}}\right]_{\rho_{\min }^{E}}^{\rho_{\max }^{E}}, \quad r \in \mathcal{N}_{i j} .
\end{aligned}
$$

Here, operator $[x]_{a}^{b}$ is defined as $[x]_{a}^{b}=\max (a, \min (x, b))$. We can observe from (20) that the allocating power depends on the priority of relay, the constraints of data rate, the transmit power and the harvest energy requirement by using $\alpha_{r}, \delta, \zeta$ and $\beta_{r}$, respectively. Similarly, $\rho_{j}^{I(r) *}$ in (21) depends on $\alpha_{r} \zeta$ and $\mu_{r}$. On the other hand, $\beta_{r}$ and $E_{j}^{R e q}$ in (22) 
force the node $j$ to increase the $\rho_{j}^{E(r)}$ to meet the constraint $C 1^{\prime}$. Moreover, (20) and (21) show that $P_{i j}^{r *}$ and $\rho_{j}^{I(r) *}$ are independent of $s_{r}$ so that we can solve $s_{r}$ separately. Relay $r$ is selected when the following criterion is satisfied

$$
s_{r}^{*}=\left\{\begin{array}{l}
1, \text { if } r=\arg \max _{k} M_{k}, \\
0, \text { otherwise },
\end{array}\right.
$$

where

$$
\begin{aligned}
M_{r}= & \frac{B}{2}\left(\alpha_{r}+\zeta\right)\left[\log _{2}\left(P_{i j}^{r *}\left(g_{i j}+g_{i r} g_{r j} \eta\left|h_{i r}\right|^{2}\right)\right)\right. \\
& +\log _{2}\left(\frac{\rho_{j}^{I(r) *}}{\rho_{j}^{I(r) *} g_{r j} \eta\left|h_{i r}\right|^{2}+g_{i r}}\right)-\frac{1}{\ln (2)} \\
& \left.-\frac{g_{i r}}{\ln (2)\left(\rho_{j}^{I(r) *} g_{r j} \eta\left|h_{i r}\right|^{2}+g_{i r}\right)}\right]-\theta,
\end{aligned}
$$

is the marginal benefit brought to the system by selecting relay $r$ in CoLink $k_{i j}$. Equation (23) means that the relay which can provide the maximum marginal benefit to the system will be selected [15]. Moreover, (23) shows that although we relax the constraint in $C 4$, the solution of $s_{r}$ is still a Boolean value. And (24) shows that the relay selection policy is determined by $\alpha_{r}$, the rate constraint, and the CSI of CoLink $k_{i j}$.

Upper Level: The Lagrange multiplier is updated. We use the gradient descent method to derive the update formula as follows

$$
\begin{aligned}
& \beta_{r}(t+1)=\left[\beta_{r}(t)-\Delta(t)\left(\eta\left|h_{i j}\right|^{2} \tilde{P}_{i j}^{r} \frac{T}{2}-\frac{E_{j}^{R e q}}{\rho_{j}^{E(r)}}\right)\right]^{+}, \\
& \delta(t+1)=\left[\delta(t)-\Delta(t)\left(P^{\max }-\sum_{r=1}^{N_{i j}} \tilde{P}_{i j}^{r}\right)\right]^{+}, \\
& \zeta(t+1)=\left[\zeta(t)-\Delta(t)\left(\sum_{r=1}^{N_{i j}} s_{r} \tilde{R}_{i j}^{r}-R^{\min }\right)\right]^{+}, \\
& \mu_{r}(t+1)=\left[\mu_{r}(t)-\Delta(t)\left(1-\tilde{\rho}_{j}^{l(r)}-\rho_{j}^{E(r)}\right)\right]^{+} .
\end{aligned}
$$

Here, operator $[x]^{+}$is defined as $[x]^{+}=\max (0, x) \cdot t$ is the iteration index and $\Delta(t)$ is the diminishing iteration step size. Note that the multiplier $\theta$ in (22)-(24) does not affect any resource allocation variables, so there is no need to update $\theta$. In next iteration, the Lagrange multipliers obtained by (25)-(28) will be used to update $\mathcal{P}, \rho, \mathcal{S}$ in lower level. The convexity of the transformed OPT-3 guarantees that the two-level iteration in (19) can finally converge to the solution of OPT-3 in (17). 
Next, we do a time complexity analysis of the proposed algorithm. As EECSR calls EESRA to solve OPT-2, we analyze EESRA first. The outer layer of EESRA implements the update of $q$, and its time complexity is $O\left(I_{\max }\right)$. Because the transformed OPT- 3 in the inner layer is proved to be convex, the complexity of solving OPT-3 for a given $q$ is $O(N)$, where $N$ is the maximum cardinality of relay candidate sets in all Colinks. So, the time complexity of EESRA is $O\left(I_{\max } \times N\right)$. In EECSR, the time complexity of the initial phase in is $O(|K|)$, the while loop runs $|K|$ times and selecting node $j$ from $Q$ requires a time complexity of $O(|K|)$ in each loop. In addition, for each input CoLink of node $j$, EECSR is called. Therefore, the time complexity of EECSR is $O\left(|K|+|K|\left(|K|+e\left(I_{\max } \times N\right)\right)\right)$, where $e$ is the maximum CoLink number of all CHs. Apparently, $e \leq|K|-1$, thus, the time complexity of EECSR is $O\left(|K|^{2} \times I_{\max } \times N\right)$.

\subsection{Distributed routing protocol}

In the previous sections, the execution node of the centralized EECSR algorithm needs to know the CSI of the entire network and the energy status of all nodes. However, this is not easy to achieve in practical networks. Therefore, inspired by the centralized EECSR, we design a table-driven distributed energy efficient cooperative SWIPT routing protocol (TDEECSR). To support TDEECSR, each CH keeps a routing table shown as Table 3. We name the first row of Table 3 as self-entry, which contains the own routing information of the node (assumed as $\mathrm{CH}_{i}$ ). The other rows are named neighbor-entries, which stores the routing information of the neighbors.

In an execution cycle, the flow of the TDEECSR protocol is as follows. The sink node first broadcasts its self-entry. Then, the neighbor $\mathrm{CHs}$ are triggered to exchange routing information (RI) with the sink. If a node updates the neighbor-entries in its routing table according to the sink's self-entry, this node will run lines 9-15 of EECSR to calculate and determine whether to update its self-entry. In the following, if any item in the selfentry of a node is updated, we call this node is activated. And once a $\mathrm{CH}$ is activated, it will compete for the channel and broadcast its self-entry to trigger neighbor nodes to exchange RI and update their routing tables. Similar to the above process, the $\mathrm{CHs}$ in the network are activated one by one until no CH's self-entry is updated. Note that any source $\mathrm{CH}$ may be activated multiple times by its neighbors and then eventually obtain its path with the smallest EEcost.

Compared to the traditional distributed Bellman-Ford routing protocol (DBF) [48, 49], TDEECSR has following advantages. First, DBF protocol requires all CHs to perform RI exchange according to a time schedule, while TDEECSR does not, which eliminates the overhead of time synchronization. Second, if the number of $\mathrm{CHs}$ in the network is $\mathrm{K}$,

Table 3 Routing table of $\mathrm{CH}_{i}$

\begin{tabular}{llllll}
\hline Node (ID) & Metrics & & & Next hope node & $\begin{array}{c}\text { Forwarding } \\
\text { energy }\end{array}$ \\
\hline self(i) & $E E \operatorname{cost}_{i}$ & $U_{(i)}$ & $U_{T P(i)}$ & Next $_{i}$ & $E_{i}^{\text {Req }}$ \\
Neighbor1 $(N 1)$ & $E E \operatorname{cost}_{N 1}$ & $U_{(N 1)}$ & $U_{T P(N 1)}$ & Next $t_{N 1}$ & $E_{N 1}^{\text {Req }}$ \\
$\ldots$ & $\ldots$ & $\ldots$ & $\ldots$ & $\ldots$ & $\ldots$ \\
Neighborj(Nj) & $E E \operatorname{cost}_{N j}$ & $U_{(N j)}$ & $U_{T P(N j)}$ & Next & $E_{N j}^{\text {Req }}$ \\
\hline
\end{tabular}


all $\mathrm{CHs}$ in DBF need to perform $\mathrm{K}$ times of RI exchange with neighbors. In TDEECSR, $\mathrm{CHs}$ are passively triggered, and they may not definitely be activated every time they are triggered. So TDEECSR avoids frequent RI exchange and thus reduces the consumption of network bandwidth and energy. Third, CHs in TDEECSR use lines 9-15 of EECSR to calculate and update their own self-entries. And this realizes dynamic sensing of neighbors' energy and real-time updating of routing metrics. In summary, TDEECSR realizes the distributed application of EECSR algorithm, and has a significant improvement in real-time performance compared to DBF. Please refer to the simulation verification in next section.

\section{Simulation results and discussion}

\subsection{Simulation setup}

We consider a network which has $30 \mathrm{CHs}$ located within a square area of $100 \mathrm{~m}^{2}$. In particular, the source $\mathrm{CH}$ is located at $(0,0)$ and the sink node is located at $(100,100)$. When laying out other nodes, we let the distance between adjacent $\mathrm{CHs}$ be within 20 $\mathrm{m}$, and the distance from cluster members to its $\mathrm{CH}$ be within $10 \mathrm{~m}$. Similar to [36], we assume that the small scale fading of links follows Rayleigh fading. So, we let $\alpha=2$ and $K_{a b}$ obey the standard Gaussian distribution. In the following experiments, unless otherwise stated, the default settings of the parameters are as follows: $B=0.2 \mathrm{MHz}$, $T=10, \eta=0.7,000000000000000 \varepsilon=2, P_{C}=0.01 \mathrm{~W}, P^{\max }=0.3 \mathrm{~W}, R^{\min }=0.1 \mathrm{Mbps}$, $\rho_{\text {min }}^{E}=\rho_{\text {min }}^{I}=0, \rho_{\text {max }}^{E}=\rho_{\text {max }}^{I}=1, E^{B}=3 \mathrm{~J}$. In addition, we uniformly set the parameters in subnetworks as follows: $N_{r}=\left|N_{i j}\right|=4, i, j \in K, \sigma_{i j}^{2}, \sigma_{i r}^{2}$ and $\sigma_{r j}^{2}$ are $-50 \mathrm{dBm}$, all the $\alpha_{r}$ equal to 1 . In the scenario of a single subnetwork, we let $E_{j}^{R e q}=0.015 \mathrm{~J}$. Generally, the value of energy efficiency is obtained by averaging 10,000 independent experimental results with different channel states.

\subsection{Convergence performance}

In this section, since the EESRA algorithm is the basis of our EECSR algorithm, we verify the convergence of the EECSR algorithm. In Fig. 3, we can observe that EESRA algorithm can converge within about 5 iterations no matter what $\sigma_{N}^{2}$ and $N_{r}$ take. Note that the value on the horizontal axis in Fig. 3 represents the iterations of the main loop in EESRA, where the iterations of the inner dual decomposition method are not counted. Figure 4 exhibits an example for the convergence process of the dual decomposition method with a given $q$.

\subsection{Impacts of parameters on energy efficiency of subnetwork transmission}

In this subsection, we show the impacts of some parameters on our energy efficiency solution for subnetwork transmission. We can observe from Fig. 3 that the optimal transmission energy efficiency of a subnet increases when the noise power decreases and the number of candidate relays increases. This result is reasonable. The reduction in noise power increases the SNR and thereby increases the channel capacity. And the increase of candidate relay number can increase the probability of selecting a more energy-efficient cooperative transmission scheme in a subnetwork. The impact of $N_{r}$ on energy efficiency can be further seen in Fig. 5 . 


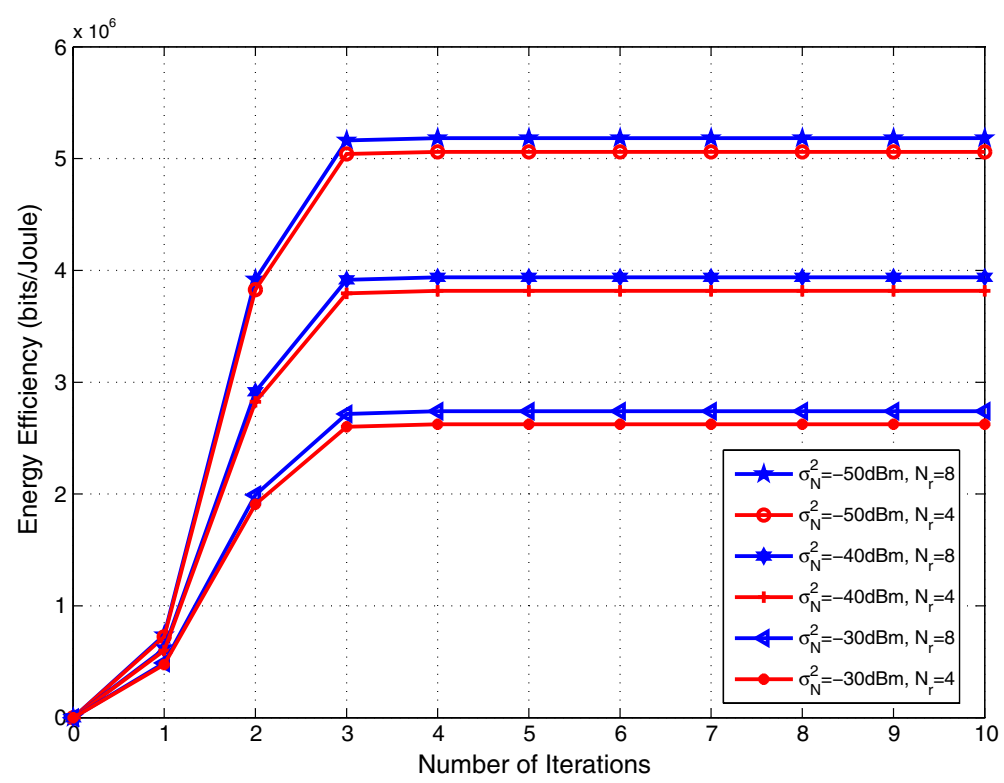

Fig. 3 Convergence performance of EESRA for $\sigma_{N}^{2}=-50 \mathrm{dBm},-40 \mathrm{dBm},-30 \mathrm{dBm}$, and $N_{r}=4,8$

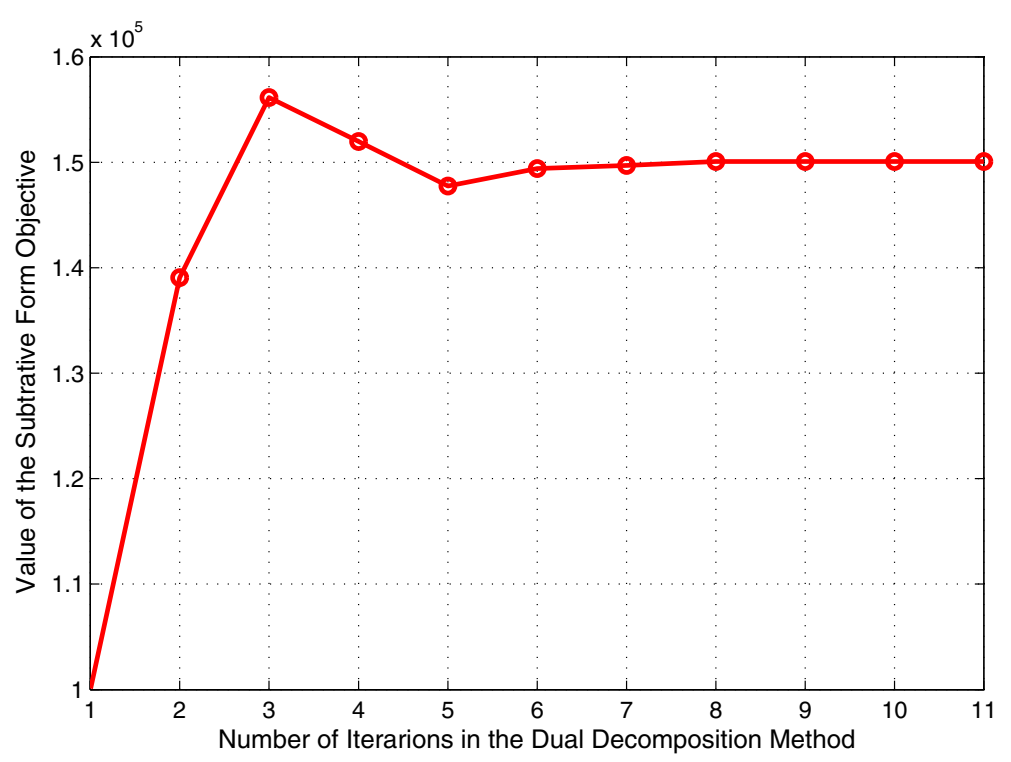

Fig. 4 Convergence of the dual decomposition method with $q=2.82 \mathrm{M}$

Figure 5 shows the optimal energy efficiency versus the number of candidate relays under direct transmission (DT) and relay transmission (RT) modes. The RT mode mentioned here corresponds to our transmission model, whose energy efficiency is solved by EESRA. The DT mode only uses the direct transmission link to implement SWIPT. For DT mode, we maximize the energy efficiency without considering cooperative relays in subnets. An important observation in Fig. 5 is that the energy efficiency of RT mode is higher than that of DT mode at the same noise level. This result illustrates that the 


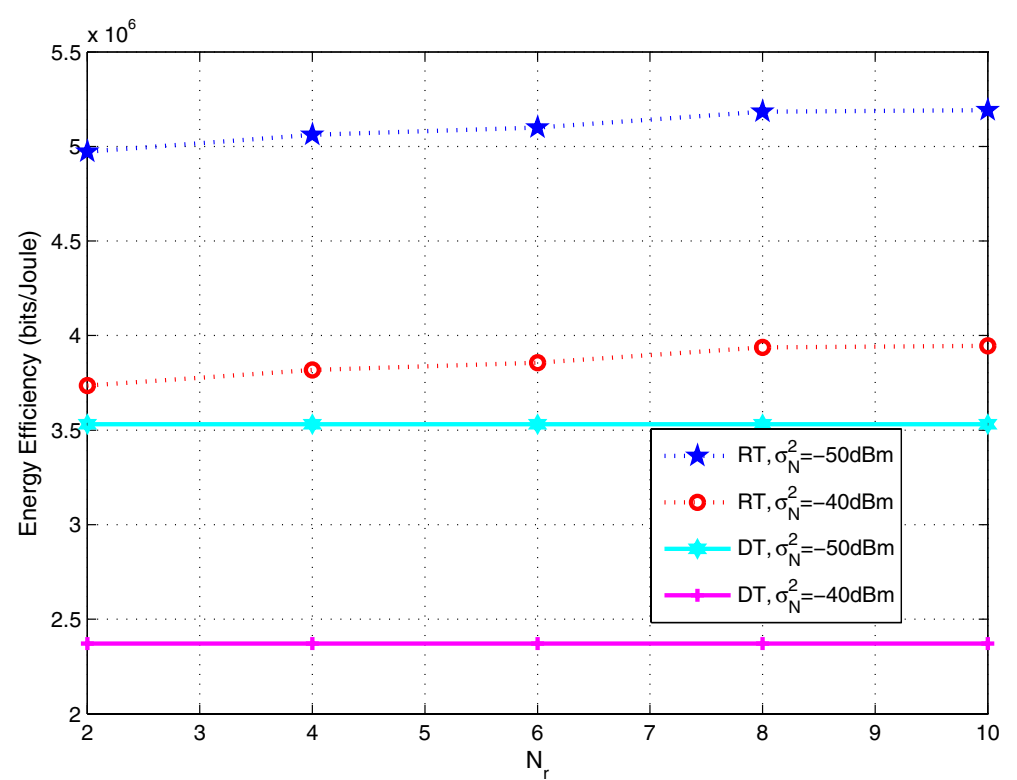

Fig. 5 Energy efficiency versus the number of relay nodes $N_{r}$ for $\sigma_{r j}^{2}=-50 \mathrm{dBm},-40 \mathrm{dBm}$ under RT and DT modes

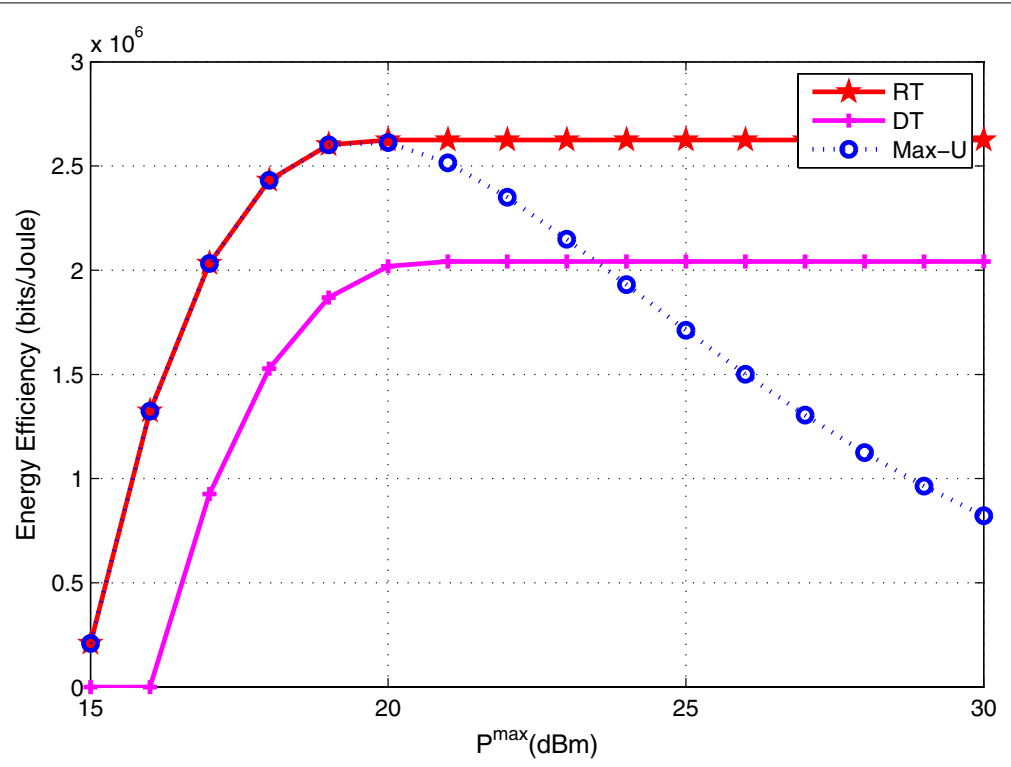

Fig. 6 Energy efficiency versus maximum transmit power allowance $P^{\max }$ in different scenarios

cooperative transmission scheme based on SWIPT-powered member relays can effectively improve the transmission energy efficiency between CHs. Figure 6 shows the energy efficiency versus $P^{\max }$ in different scenarios. As $P^{\max }$ gradually increases from a small value, the energy efficiency in RT and DT modes increase rapidly until $P^{\text {max }}$ is larger than $20 \mathrm{dBm}$. This is because that once the maximum efficiency is achieved, the increase in transmit power will result in a decrease in energy efficiency. In order to show this phenomenon, we change the optimization goal of OPT-2 to maximize the 
throughput and solve it with EESRA. The obtained energy efficiency curve is shown by the blue dotted line with the name Max-U in Fig. 6.

\subsection{Performance verification of the routing algorithm}

\subsubsection{Path energy efficiency comparison for different transmission modes}

In this subsection, Fig. 7 depicts the path energy efficiency versus minimum data rate requirement $R^{\text {min }}$ under DT and RT modes. Obviously, the path energy efficiency obtained under RT mode is higher than that under DT mode. As $R^{\text {min }}$ gradually increases, the path energy efficiency first remains constant within a range, then slightly increases, and finally decreases gradually. The reason is that when $R^{\text {min }}$ reaches a certain value, the nodes in the path have to increase their transmit power or $\rho^{I}$ to meet the rate requirement, and thus improve the energy efficiency. But as the transmit power continues to increase, the energy efficiency in each subnetwork eventually decreases. From Fig. 7, we can draw another conclusion that on the premise of no decreasing energy efficiency, the achievable maximum $R^{\text {min }}$ of RT mode is greater than that of DT mode.

\subsubsection{Performance verification of the SWIPT routing algorithm}

In this subsection, we first give an example shown in Figs. 8 and 9 to explain the benefit of EECSR algorithm. In Figs. 8 and 9, a CoLink between two CHs is represented by a dotted line for simplicity. In Fig. 8, all CHs work in the information transmission mode (denoted as $\mathrm{CH}-\mathrm{IT}$ ). To find the transmission path in $\mathrm{CH}$-IT mode, we use an algorithm which is similar to EECSR with the $E_{j}^{R e q}=0, \rho_{j}^{I(r)}=1$, and $\rho_{j}^{E(r)}=0$. In the case that the energy of $\mathrm{CH}_{8}$ is insufficient for forwarding, the energy efficiency of the solution path from the source $\mathrm{CH}_{2}$ to the sink is $0.44 \mathrm{Mbits} / \mathrm{J}$, and the hop number of the path is 13 . By contrast, in Fig. 9, where all CHs support the SWIPT transmission mode (denoted as $\mathrm{CH}$-SWIPT), the energy efficiency of the path obtained by EECSR is $0.57 \mathrm{Mbits} / \mathrm{J}$, and

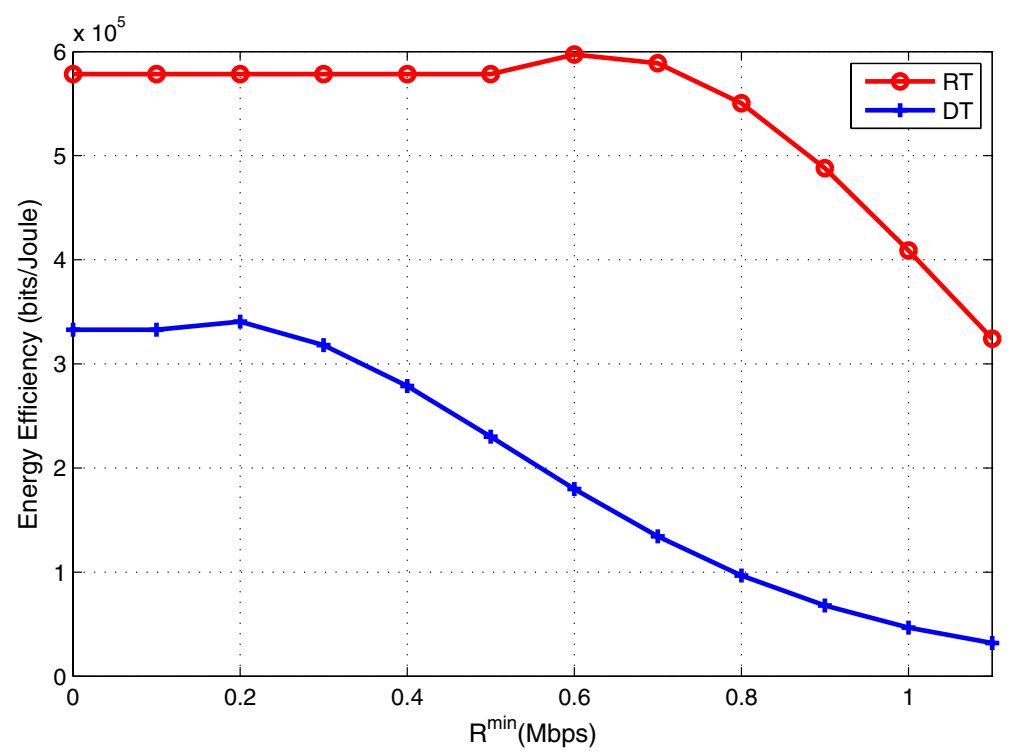

Fig. 7 Path energy efficiency versus minimum data rate requirement $R^{\text {min }}$ 


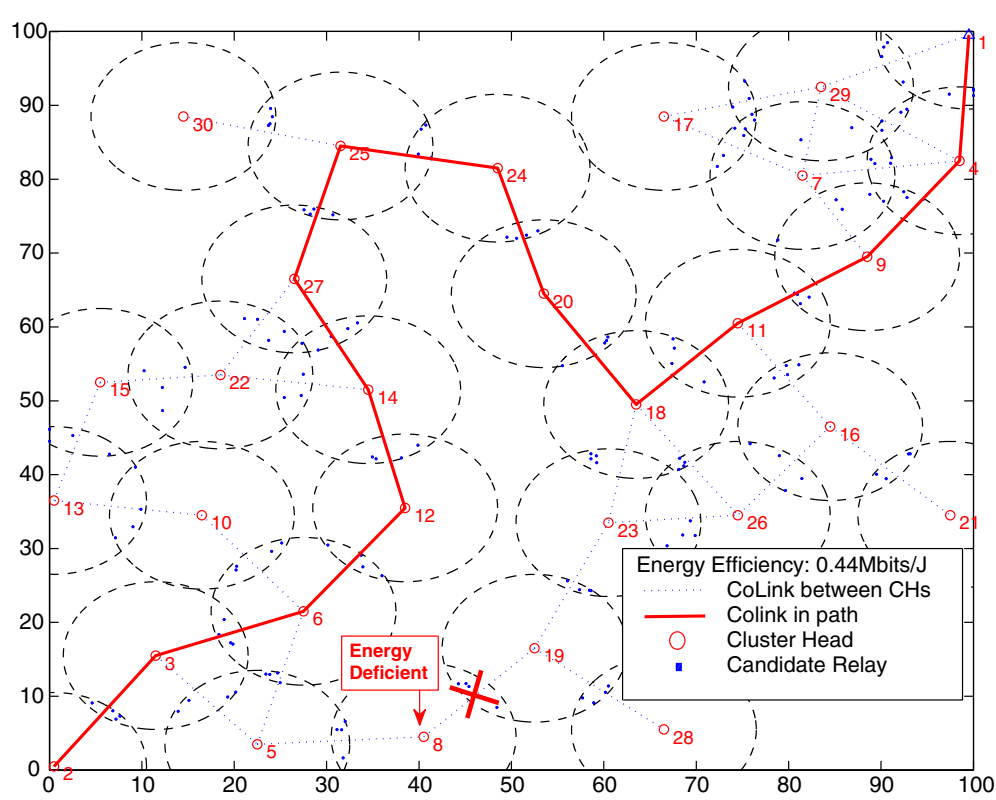

Fig. 8 An example for Path solution under $\mathrm{CH}-\mathrm{IT}$ mode

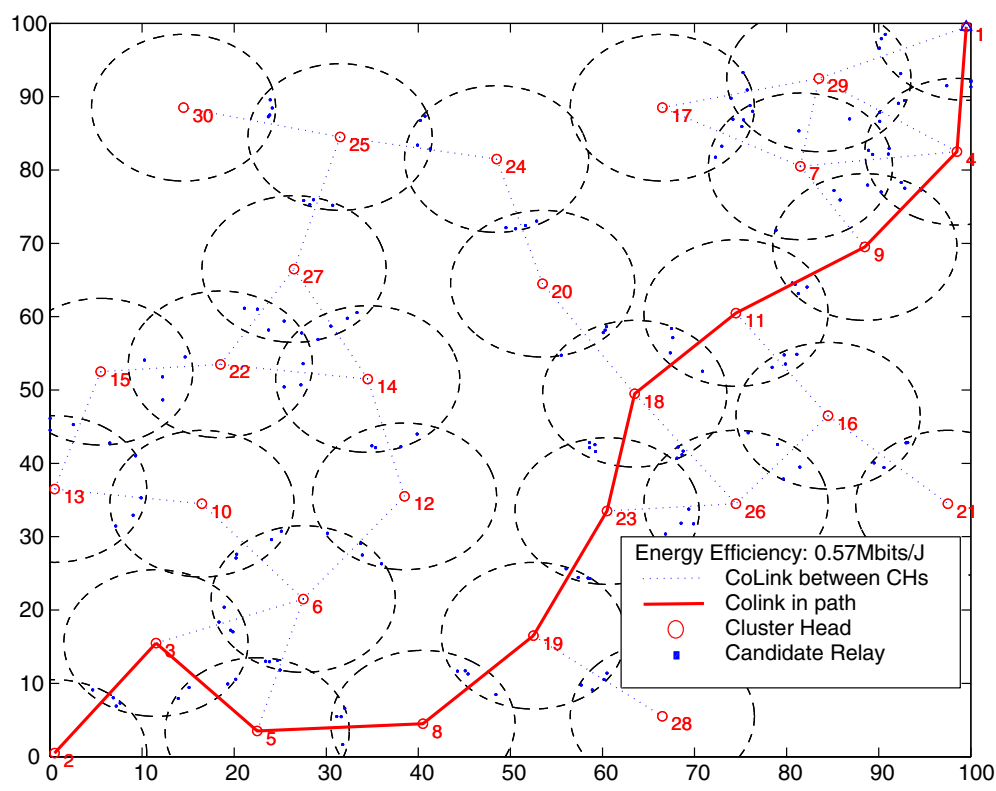

Fig. 9 An example for Path solution of EECSR under CH-SWIPT mode

the hop number is 10 . This illustrates that the algorithm combining SWIPT and routing may find a more energy-efficient transmission path.

Next, we give Fig. 10 to show the average feasible energy efficiency versus the energydeficient node ratio (EDNR), For each fixed EDNR, we perform $N$ experiments, and in each experiment, we randomly set the corresponding number of $\mathrm{CHs}$ as energy-deficient according to the EDNR. The average feasible energy efficiency is defined as 


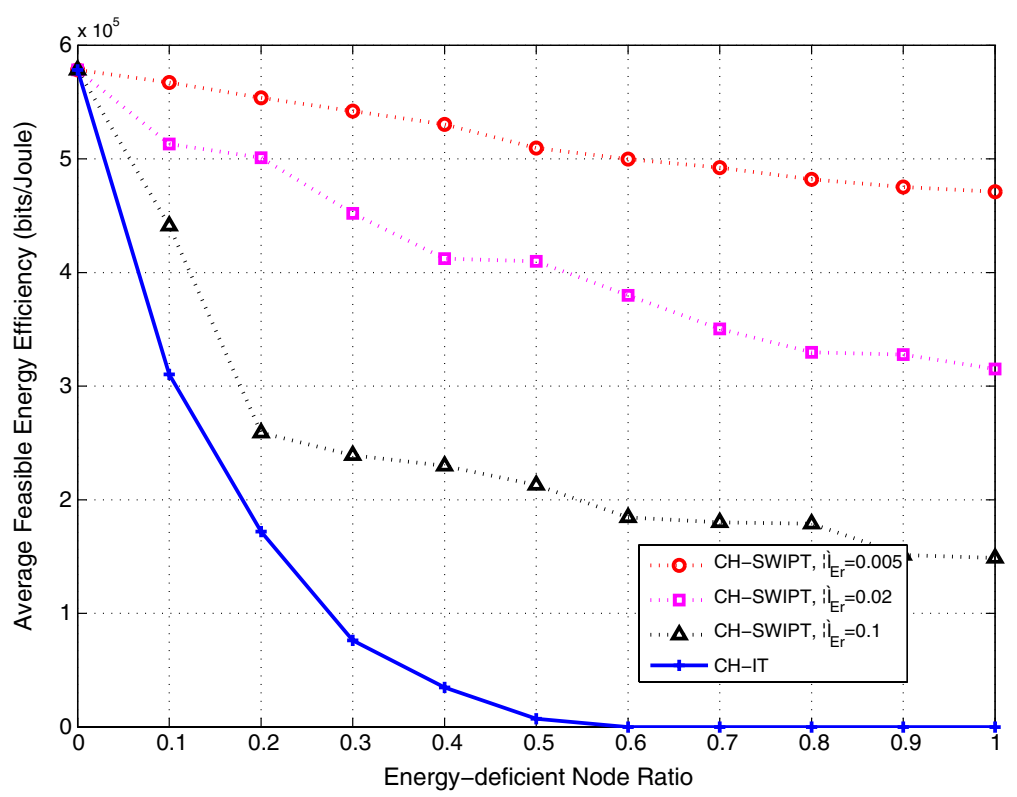

Fig. 10 Average feasible energy efficiency versus the energy-deficient node ratio

AvUeff $=\left(\sum_{l=1}^{M}\right.$ PUeff $) / N$, where $M$ is the number of experiments that can successfully find a feasible path, and PUeffl is the energy efficiency of a feasible path. In Fig. 10, the blue solid line shows the change of $A v U$ eff in $\mathrm{CH}$-IT mode. In CH-SWIPT mode, the value of $E^{R e q}$ of each energy-deficient $\mathrm{CH}$ obeys the normal distribution $C N\left(\mu_{E r}, \sigma_{E r}^{2}\right)$, where $\mu_{E r}$ and $\sigma_{E r}^{2}$ are the mean and variance, respectively. The three dotted lines in Fig. 10 are the corresponding AvUeff curves with different $\mu_{E r}$. Compared with $\mathrm{CH}$-IT mode, the path obtained by EECRT based on CH-SWIPT mode is more energy efficient. In addition, as EDNR increases, the $A v U$ eff in CH-IT mode decreases significantly. This is because $M$ in $A v U$ Ueff decreases rapidly with node failure. In comparison, the value of AvUeff in CH-SWIPT mode drops much slower, which reveals the robustness of our EECSR algorithm.

\subsection{Comparison on time delay for routing protocols}

In this section, we compare the time delay of routing protocols, which are the protocol based on the EECSR, TDEECSR and the protocol based on the DBF algorithm (abbreviated as DBFR). To ensure comparability, all simulations run on the same computer with the following configuration: intel core i7-8750H-2.20G processor, $16 \mathrm{BG}$ DDR4-2666 MHz memory, and GeForce GTX 1060-6 GB graphics card. Figure 11 depicts the time delay caused by the route discovery process of protocol (in logarithmic scale) versus $\mathrm{CH}$ number. Note that TDEECSR-Av and DBFR-Av curves represent the average time spent on each $\mathrm{CH}$ by the two corresponding distributed routing protocols, respectively. It can be observed that the time consumption of the three protocols increases with the number of $\mathrm{CH}$ in the network. EECSR takes the least time and DBFR takes the most. TDEECSRP takes significantly less time than DBFRP, and takes 5-20\% longer time than CEESRP. And the average time of TDEECSRP is significantly less than the concentrated time of EESCRP, which shows 


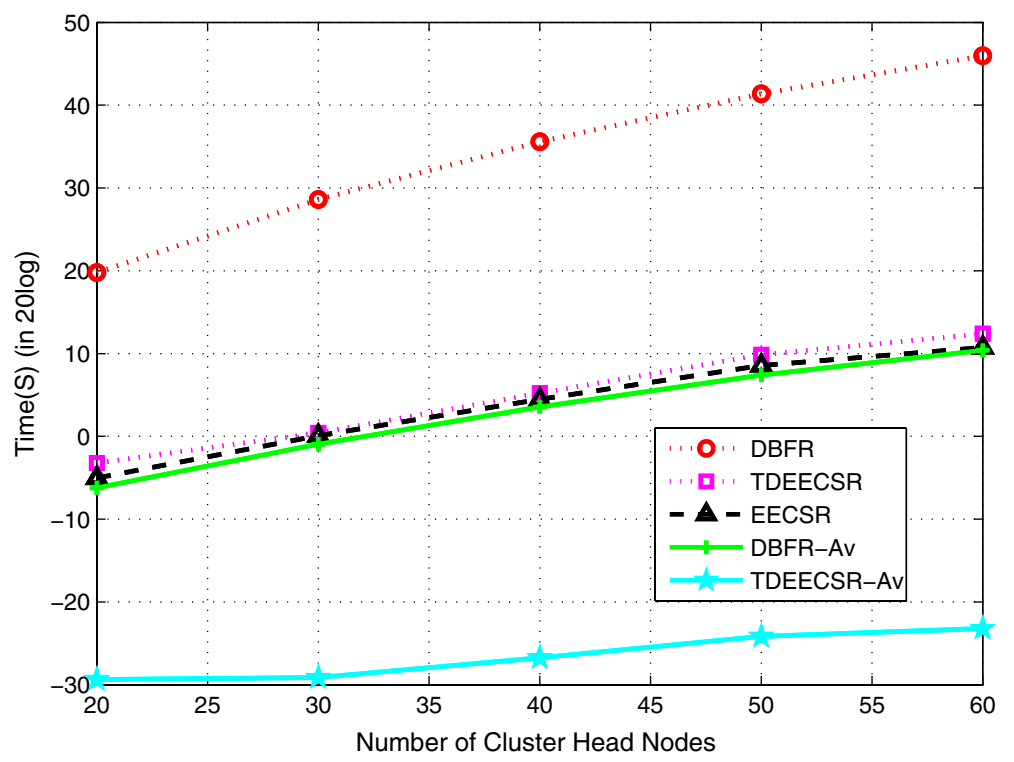

Fig. 11 Time delay of route discovery (in logarithmic scale) versus $\mathrm{CH}$ number

that TDEECSRP not only distributes the workload of the node like DBFRP, but also has the approximate total time level of EECSRP. Therefore, TDEECSRP is more suitable for larger real-time sensor networks than the other two protocols.

\section{Conclusions}

In this paper, we have considered applying SWIPT and routing selection to multi-hop clustered WSNs, where member relays between two $\mathrm{CHs}$ have used the harvested energy to achieve cooperative transmission, while $\mathrm{CH}$ relays have utilized SWIPT to supplement battery energy and forward information. We have aimed to find optimal policies, which includes routing policy for $\mathrm{CHs}$, transmission power allocation, harvest energy splitting and member relays selection, and to find the maximum energy efficiency transmission path from any source $\mathrm{CH}$ to the sink. To achieve this goal, we have proposed a heuristic energy efficient cooperative SWIPT routing algorithm that consists of an outer energy efficient routing algorithm and multiple inner resource allocation algorithms to obtain the path with maximum energy efficiency and the resource allocation policies for each hop. Based on our routing algorithm, we have designed a table-driven distributed energy efficient cooperative SWIPT routing protocol to support distributed applications. Simulation results have shown that our SWIPT routing algorithm can achieve significant energy efficiency and good robustness. And our distributed routing protocol has been verified to have better real-time performance than traditional ones.

\section{Appendix}

Proof of convexity of the transformed OPT-3 problem

Similar to [15], we prove the convexity of the transformed OPT-3 problem. We first prove the concavity of $F(\mathcal{P}, \rho, \mathcal{S})$ in (16) for a given $q$. We define a function 
$f_{r}\left(\boldsymbol{x}_{\boldsymbol{r}}\right)=\frac{B}{2} \alpha_{r} \log _{2}\left(\frac{\tilde{P}_{i j}^{r} \tilde{\rho}_{j}^{(r)}\left(g_{i j}+g_{r i} \eta\left|h_{i r}\right|^{2}\right) g_{i r}}{g_{i r}+\tilde{\rho}_{j}^{I(r)} g_{r j} \eta\left|h_{i r}\right|^{2}}\right)$ whose input is $\boldsymbol{x}_{\boldsymbol{r}}=\left[\tilde{P}_{i j}^{r}, \tilde{\rho}_{j}^{I(r)}, \rho_{j}^{E(r)}\right]$. Then the Hessian matrix of function $f_{r}\left(\boldsymbol{x}_{r}\right)$ is given by

$$
\mathbf{H}\left(f_{r}\left(\boldsymbol{x}_{\boldsymbol{r}}\right)\right)=\left[\begin{array}{ccc}
\tau_{1} & 0 & 0 \\
0 & \tau_{2} & 0 \\
0 & 0 & \tau_{3}
\end{array}\right],
$$

where $\tau_{1}, \tau_{2}$ and $\tau_{3}$ are the eigenvalues of $\mathbf{H}\left(f_{r}\left(\boldsymbol{x}_{\boldsymbol{r}}\right)\right)$. Specifically, we can derive that $\tau_{1}=-\frac{B \alpha_{r}}{2 \ln (2)\left(\tilde{P}_{i j}^{r}\right)^{2}}, \quad \tau_{2}=-\frac{B \alpha_{r} g_{i r}\left(g_{i r}+2 \widetilde{\rho}_{j}^{I(r)} g_{r j} \eta\left|h_{i r}\right|^{2}\right)}{2 \ln (2)\left[\tilde{\rho}_{j}^{I(r)}\left(g_{i r}+\widetilde{\rho}_{j}^{I(r)} g_{r j} \eta\left|h_{i r}\right|^{2}\right)\right]^{2}}, \quad \tau_{3}=0 . \quad$ Since $\tau_{i} \leq 0, i=1,2,3$, the hessian matrix of $f_{r}\left(\boldsymbol{x}_{\boldsymbol{r}}\right)$ is semi-definite, which deduces that $f_{r}\left(\boldsymbol{x}_{\boldsymbol{r}}\right)$ is jointly concave with respect to (w.r.t.) $\tilde{P}_{i j}^{r}, \tilde{\rho}_{j}^{I(r)}, \rho_{j}^{E(r)}$. Then, we convert $f_{r}\left(\boldsymbol{x}_{\boldsymbol{r}}\right)$ into $u_{r}\left(\boldsymbol{x}_{r}\right)$ by using perspective transformation that can maintain convexity [46], where $u_{r}\left(\boldsymbol{x}_{r}\right)=s_{r} f_{r}\left(\boldsymbol{x}_{r} / s_{r}\right)$. So, $u_{r}\left(\boldsymbol{x}_{r}\right)$ is jointly concave w.r.t. $\tilde{P}_{i j}^{r}, \tilde{\rho}_{j}^{I(r)}, \rho_{j}^{E(r)}$ and $s_{r}$. Furthermore, it can be observed that the non-negative weighted sum of $u_{r}\left(\boldsymbol{x}_{r}\right)$ can form $U_{(i j)}(\mathcal{P}, \boldsymbol{\rho}, \mathcal{S})$. Thus, concavity of $U_{(i j)}(\mathcal{P}, \rho, \mathcal{S})$ is proved. Moreover, $U_{T P(i j)}(\mathcal{P}, \boldsymbol{\rho}, \mathcal{S})$ is an affine function relative to the optimization variables. Therefore, $F(\mathcal{P}, \rho, \mathcal{S})$ is jointly concave w.r.t. $\tilde{P}_{i j}^{r}, \tilde{\rho}_{j}^{I(r)}, \rho_{j}^{E(r)}$ and $s_{r}$.

Second, we explain the convexity of constraints $C 1^{\prime}-C 8$. It can be seen that the inequality in $C 1^{\prime}$ is composed of linear terms and convex terms, thus $C 1^{\prime}$ is convex. $C 2$ is linear function of $\tilde{P}_{i j}^{r}$. $C 3$ is convex according to $U_{(i j)}(\mathcal{P}, \rho, \mathcal{S})$. The relaxed constraint $C 4$ and constraints $C 7-C 10$ are linear inequalities. As a result, the transformed OPT-3 problem with constraints $C 1^{\prime}-C 8$ is convex with respect to $\tilde{P}_{i j}^{r}, \tilde{\rho}_{j}^{I(r)}, \rho_{j}^{E(r)}$ and $s_{r}$.

\section{Abbreviations}

SWIPT:: Simultaneous wireless information and power transfer; MCWSN:: Multi-hop clustered wireless sensor networks; RF:: Radio frequency; EECSR:: Energy efficient cooperative SWIPT routing; WSN:: Wireless sensor network; PS:: Power splitting; EH:: Energy harvester; DF:: Decode-and-forward; SNR:: The signal to noise ratio; MRC:: Maximum ratio combining; QoS:: Quality of service; EESRA:: Energy efficient subnet resource allocation algorithm; KKT:: Karush-Kuhn-Tucker; TDEECSR:: Table-driven distributed energy-efficient cooperative SWIPT routing; RI:: Routing information; DBF:: Distributed Bellman-Ford; EDNR:: Energy-deficient node ratio; RAN:: Radio access network.

\section{Acknowledgements}

This work has been supported by the Natural Science Foundation of China under Grant 61501069 and the special project of technological innovation and application development of Chongqing(NO.cstc2019jscx-msxmX0167).

\section{Authors' contributions}

Han conceived the study and participated in most of the design work and implementation. Liu and Huang participated in algorithm design. Wang and Zhong participated in the experimental design and network model design. All authors read and approved the final manuscript.

\section{Funding}

This work was supported in part by the Natural Science Foundation of China under Grant 61501069.

Availability of data and materials

Data sharing is not applicable to this article as no datasets were generated or analyzed during the current study.

Declarations

\section{Competing interests}

The authors declare that they have no competing interests.

\section{Author details}

${ }^{1}$ School of Microelectronics and Communication Engineering, Chongqing University, Chongqing 400044, China. ${ }^{2}$ College of Computer Science, Chongqing University, Chongqing 400044, China. ${ }^{3}$ College of Electronic and Information Engineering, Southwest University, Chongqing 400715, China. 
Received: 21 December 2020 Accepted: 23 February 2021

Published online: 09 March 2021

\section{References}

1. I.S.H. Martinez, I.P.O.J. Salcedo, I.B.S.R. Daza, IoT application of WSN on 5 G infrastructure. In: 2017 International Symposium on Networks, Computers and Communications, ISNCC 2017 (2017). https://doi.org/10.1109/ISNCC .2017 .8071989

2. K. Shafique, B.A. Khawaja, F. Sabir, S. Qazi, M. Mustaqim, Internet of things (IoT) for next-generation smart systems: a review of current challenges, future trends and prospects for emerging 5G-IOT scenarios. IEEE Access $\mathbf{8}, 23022$ 23040 (2020). https://doi.org/10.1109/ACCESS.2020.2970118

3. E.B. Ali, S. Kishk, E.H. Abdelhay, Multidimensional auction for task allocation using computation offloading in fifth generation networks. Future Gener. Comput. Syst. 108, 717-725 (2020). https://doi.org/10.1016/j.future.2020.02.021

4. H.C. Hsieh, J.L. Chen, A. Benslimane, $5 \mathrm{G}$ virtualized multi-access edge computing platform for loT applications. J. Netw. Comput. Appl. 115, 94-102 (2018). https://doi.org/10.1016/j.jnca.2018.05.001

5. A. Mavromatis, C. Colman-Meixner, A.P. Silva, X. Vasilakos, R. Nejabati, D. Simeonidou, A software-defined loT device management framework for edge and cloud computing. IEEE Internet Things J. 7(3), 1718-1735 (2020). https://doi. org/10.1109/JIOT.2019.2949629

6. S. Tyagi, S. Tanwar, S.K. Gupta, N. Kumar, J.J.P.C. Rodrigues, A lifetime extended multi-levels heterogeneous routing protocol for wireless sensor networks. Telecommun. Syst. 59(1), 43-62 (2015). https://doi.org/10.1007/s1123 5-014-9884-5

7. Z. Zhu, W. Ge, N. Wang, Y. Wang, W. Hao, Z. Chu, Z. Wang, AN-Based beamforming design in secrecy heterogeneous WSN with MIMO-SWIPT. In: 2019 IEEE International Conference on Communications Workshops, ICC Workshops 2019_Proceedings (2019). https://doi.org/10.1109/ICCW.2019.8756686

8. G. Hatzivasilis, K. Fysarakis, O. Soultatos, I. Askoxylakis, I. Papaefstathiou, G. Demetriou, The industrial internet of things as an enabler for a circular economy Hy-LP: a novel IloT protocol, evaluated on a wind park's SDN/NFV-enabled 5G industrial network. Comput. Commun. 119, 127-137 (2018). https://doi.org/10.1016/j.comcom.2018.02.007

9. C. Delgado, M. Canales, J. Ortin, J.R. Gallego, A. Redondi, S. Bousnina, M. Cesana, Joint application admission control and network slicing in virtual sensor networks. IEEE Internet Things J. 5(1), 28-43 (2018). https://doi.org/10.1109/ JIOT.2017.2769446

10. A. Napolitano, A. Giorgetti, K. Kondepu, L. Valcarenghi, P. Castoldi, Network slicing: an overview. In: IEEE 4th International Forum on Research and Technologies for Society and Industry, RTSI 2018_Proceedings (2018). https://doi. org/10.1109/RTSI.2018.8548449

11. Y. Sun, S. Qin, G. Feng, L. Zhang, M. Imran, Service provisioning framework for RAN slicing: user admissibility, slice association and bandwidth allocation. IEEE Trans. Mob. Comput. (2020). https://doi.org/10.1109/tmc.2020.3000657

12. Y.-J. Liu, G. Feng, Y. Sun, S. Qin, Y.-C. Liang, Device association for RAN slicing based on hybrid federated deep reinforcement learning. IEEE Trans. Veh. Technol. 69(12), 15731-15745 (2020). https://doi.org/10.1109/tvt.2020.3033035

13. Y. Sun, W. Jiang, G. Feng, P.V. Klaine, L. Zhang, M.A. Imran, Y.C. Liang, Efficient handover mechanism for radio access network slicing by exploiting distributed learning. IEEE Trans. Netw. Serv. Manag. 17(4), 2620-2633 (2020). https:// doi.org/10.1109/TNSM.2020.3031079

14. L. Dash, M. Khuntia, Energy efficient techniques for 5 G mobile networks in WSN: a Survey. In: 2020 International Conference on Computer Science, Engineering and Applications, ICCSEA 2020 (2020). https://doi.org/10.1109/ICCSE A49143.2020.9132941

15. D.W.K. Ng, E.S. Lo, R. Schober, Wireless information and power transfer: energy efficiency optimization in OFDMA systems. IEEE Trans. Wirel. Commun. 12(12), 6352-6370 (2013). https://doi.org/10.1109/TWC.2013.103113.130470

16. M. Sheng, L. Wang, X. Wang, Y. Zhang, C. Xu, J. Li, Energy efficient beamforming in MISO heterogeneous cellular networks with wireless information and power transfer. IEEE J. Sel. Areas Commun. 34(4), 954-968 (2016). https:// doi.org/10.1109/JSAC.2016.2544538

17. X. Liu, Y. Jia, Z. Wen, J. Zou, S. Li, Beamforming design for full-duplex SWIPT with co-channel interference in wireless sensor systems. Sensors 18(10), 3362 (2018). https://doi.org/10.3390/s18103362

18. R. Zhang, C.K. Ho, MIMO broadcasting for simultaneous wireless information and power transfer. IEEE Trans. Wirel. Commun. 12(5), 1989-2001 (2013). https://doi.org/10.1109/TWC.2013.031813.120224

19. L. Liu, R. Zhang, K.C. Chua, Wireless information and power transfer: a dynamic power splitting approach. IEEE Trans. Commun. 61(9), 3990-4001 (2013). https://doi.org/10.1109/TCOMM.2013.071813.130105

20. D.W.K. Ng, E.S. Lo, R. Schober, Energy-efficient resource allocation in multiuser OFDM systems with wireless information and power transfer. IEEE Wirel. Commun. Netw. Conf. WCNC (2013). https://doi.org/10.1109/WCNC.2013.65551 84

21. D.W.K. Ng, E.S. Lo, R. Schober, Energy-efficient power allocation in OFDM systems with wireless information and power transfer. IEEE Int. Conf. Commun. (2013). https://doi.org/10.1109/ICC.2013.6655208

22. S. Guo, Y. Shi, Y. Yang, B. Xiao, Energy Efficiency Maximization in Mobile Wireless Energy Harvesting Sensor Networks. IEEE Transactions on Mobile Computing 17(7), 1524-1537 (2018). https://doi.org/10.1109/TMC.2017.2773067

23. S. Guo, X. Zhou, X. Zhou, Energy-efficient resource allocation in SWIPT cooperative wireless networks. IEEE Syst. J. 14(3), 4131-4142 (2020). https://doi.org/10.1109/JSYST.2019.2961001

24. X. Zhou, Q. Li, Energy efficiency for SWIPT in MIMO two-way amplify-and-forward relay networks. IEEE Trans. Veh. Technol. 67(6), 4910-4924 (2018). https://doi.org/10.1109/TVT.2018.2819682

25. Q. Li, L. Yang, Robust optimization for energy efficiency in MIMO two-way relay networks with SWIPT. IEEE Syst. J. 14(1), 196-207 (2020). https://doi.org/10.1109/JSYST.2019.2904721

26. Y. Zhang, J. He, S. Guo, F. Wang, Energy efficiency maximisation in wireless powered networks with cooperative nonorthogonal multiple access. IET Commun. 12(18), 2374-2383 (2018). https://doi.org/10.1049/iet-com.2018.5056 
27. Y. Yuan, Y. Xu, Z. Yang, P. Xu, Z. Ding, Energy efficiency optimization in full-duplex user-aided cooperative SWIPT NOMA systems. IEEE Trans. Commun. 67(8), 5753-5767 (2019). https://doi.org/10.1109/tcomm.2019.2914386

28. D.S. Michalopoulos, H.A. Suraweera, R. Schober, Relay selection for simultaneous information transmission and wireless energy transfer: a tradeoff perspective. IEEE J. Sel. Areas Commun. 33(8), 1578-1594 (2015). https://doi. org/10.1109/JSAC.2015.2391771

29. T. Liu, X. Wang, L. Zheng, Cooperative SWIPT scheme for wirelessly powered sensor networks. IEEE Trans. Commun. 65(6), 2740-2752 (2017). https://doi.org/10.1109/TCOMM.2017.2685580

30. K. Shin, H. Lee, D.H. Cho, DiBA: distributed bottleneck alleviation scheme in wireless multi-hop sensor networks. IEEE Commun. Lett. 18(3), 431-434 (2014). https://doi.org/10.1109/LCOMM.2013.123113.131516

31. Y. Rong, Y. Hua, Optimality of diagonalization of multi-hop MIMO relays. IEEE Trans. Wirel. Commun. 8(12), 6068-6077 (2009). https://doi.org/10.1109/TWC.2009.12.090379

32. P. Sethu Lakshmi, M.G. Jibukumar, Performance analysis of SWIPT in multi-hop wireless sensor networks. Procedia Comput. Sci. 171, 2157-2166 (2020). https://doi.org/10.1016/j.procs.2020.04.233

33. D.K.P. Asiedu, H. Lee, K.J. Lee, Transmit power minimization for a multi-hop SWIPT decode-and-forward sensor network. IEEE Veh. Technol. Conf. (2019). https://doi.org/10.1109/NTCFall.2019.8891203

34. X. Liu, Z. Wen, D. Liu, J. Zou, S. Li, Joint source and relay beamforming design in wireless multi-hop sensor networks with SWIPT. Sensors (Switzerland) (2019). https://doi.org/10.3390/s19010182

35. Q. Wu, X. Zhou, Q. Cao, H. Fang, Multihop capability analysis in wireless information and power transfer multirelay cooperative networks. Wirel. Commun. Mob. Comput. (2018). https://doi.org/10.1155/2018/1857015

36. S. Guo, F. Wang, Y. Yang, B. Xiao, Energy-efficient cooperative Tfor simultaneous wireless information and power transfer in clustered wireless sensor networks. IEEE Trans. Commun. 63(11), 4405-4417 (2015). https://doi. org/10.1109/TCOMM.2015.2478782

37. S. He, Y.Tang, Z. Li, F. Li, K. Xie, H.J. Kim, G.J. Kim, Interference-aware routing for difficult wireless sensor network environment with SWIPT. Sensors (Switzerland) (2019). https://doi.org/10.3390/s19183978

38. S. He, K. Xie, W. Chen, D. Zhang, J. Wen, Energy-aware routing for SWIPT in multi-hop energy-constrained wireless network. IEEE Access 6, 17996-18008 (2018). https://doi.org/10.1109/ACCESS.2018.2820093

39. N.A. Pantazis, S.A. Nikolidakis, D.D. Vergados, Energy-efficient routing protocols in wireless sensor networks: a survey. IEEE Commun. Surv. Tutor. 15(2), 551-591 (2013). https://doi.org/10.1109/SURV.2012.062612.00084

40. K. Thakkar Mansi, M.M. Patel, Energy efficient routing in wireless sensor network. Proc. Int. Conf. Invent. Res. Comput. Appl. ICIRCA 2018, 264-268 (2018). https://doi.org/10.1109/ICIRCA.2018.8597353

41. A.A. Nasir, D.T. Ngo, X. Zhou, R.A. Kennedy, S. Durrani, Joint resource optimization for multicell networks with wireless energy harvesting relays. IEEE Trans. Veh. Technol. 65(8), 6168-6183 (2016). https://doi.org/10.1109/ TVT.2015.2472295

42. K. Xie, X. Wang, X. Liu, J. Wen, J. Cao, Interference-aware cooperative communication in multi-radio multi-channel wireless networks. IEEE Trans. Comput. 65(5), 1528-1542 (2016). https://doi.org/10.1109/TC.2015.2448089

43. W. Dinkelbach, On nonlinear fractional programming. Manag Sci 13, 492-498 (1967). https://doi.org/10.1287/ mnsc.13.7.492

44. D.W.K. Ng, E.S. Lo, R. Schober, Energy-efficient resource allocation in OFDMA systems with large numbers of base station antennas. IEEE Trans. Wirel. Commun. 11(9), 3292-3304 (2012). https://doi.org/10.1109/TWC.2012.07251 2.111850

45. W. Yu, R. Lui, Dual methods for nonconvex spectrum optimization of multicarrier systems. IEEE Trans. Commun. 54(7), 1310-1322 (2006). https://doi.org/10.1109/TCOMM.2006.877962

46. S. Boyd, L. Vandenberghe, Convex Optimization, p. 100. Cambridge University Press, Cambridge (2004). https://doi. org/10.1017/cbo9780511804441. https://www.cambridge.org/core/product/identifier/9780511804441/type/book

47. K. Seong, M. Mohseni, J.M. Cioffi, Optimal resource allocation for OFDMA downlink systems. In: IEEE International Symposium on Information Theory_-Proceedings, pp. 1394-1398 (2006). https://doi.org/10.1109/ISIT.2006.262075

48. B. Awerbuch, A. Bar-Noy, M. Gopal, Approximate distributed Bellman-Ford algorithms. Proc. IEEE INFOCOM 3, 1206-1213 (1991)

49. M.L. Daggitt, A.J.T. Gurney, T.G. Griffin, Asynchronous convergence of policy-rich distributed Bellman-Ford routing protocols. In: SIGCOMM 2018-Proceedings of the 2018 Conference of the ACM Special Interest Group on Data Communication, pp. 103-116 (2018). https://doi.org/10.1145/3230543.3230561

\section{Publisher's Note}

Springer Nature remains neutral with regard to jurisdictional claims in published maps and institutional affiliations. 